\title{
Monte Carlo Results for Projected Self-Avoiding Polygons: A Two-dimensional Model for Knotted Polymers
}

\author{
E. Guitter and E. Orlandini \\ CEA-Saclay, Service de Physique Théorique, \\ F-91191 Gif-sur-Yvette Cedex, France
}

October 3, 2018

\begin{abstract}
We introduce a two-dimensional lattice model for the description of knotted polymer rings. A polymer configuration is modeled by a closed polygon drawn on the square diagonal lattice, with possible crossings describing pairs of strands of polymer passing on top of each other. Each polygon configuration can be viewed as the two-dimensional projection of a particular knot. We study numerically the statistics of large polygons with a fixed knot type, using a generalization of the BFACF algorithm for self-avoiding walks. This new algorithm incorporates both the displacement of crossings and the three types of Reidemeister transformations preserving the knot topology. Its ergodicity within a fixed knot type is not proven here rigorously but strong arguments in favor of this ergodicity are given together with a tentative sketch of proof. Assuming this ergodicity, we obtain numerically the following results for the statistics of knotted polygons: In the limit of a low crossing fugacity, we find a localization along the polygon of all the primary factors forming the knot. Increasing the crossing fugacity gives rise to a transition from a self-avoiding walk to a branched polymer behavior.
\end{abstract}


The statistical properties of self-avoiding polymers are now very well understood, mainly thanks to the famous equivalence of the problem to a field theory, leading to a natural derivation of scaling laws and critical exponents [1]. In three dimensions however, closed polymers will generally form knots, which, for self-avoiding objects, will restrict the accessible configurations. In the last few years, some progress has been made towards answering some basic questions about knots, showing for instance that a sufficiently long polymer is knotted with probability one [2, 3], or computing the distribution of random knots [ [由]. However, the statistics of a closed polymer with a fixed knot-type is less well understood. Indeed, the usual field theory approach does not account for this distinction of the knot type, but corresponds rather to a summation over all the possible knot topologies. In particular, the natural question of how the critical exponents depend on the knot topology remains an open issue. Knotted configurations are encountered in the description of closed DNA molecules, with an apparent influence of the knot type on some of their properties [5].

An attempt to understand the role of a fixed knot topology was done in [6, 7,8 , 9, 10, 11] where a numerical study of knotted lattice polygons in the cubic lattice $Z^{3}$ was performed. There it was shown that, while the connectivity constant and the size exponent $\nu$ for the radius of gyration are independent of the knot type, the entropic exponent $\alpha$ for the number of accessible configurations clearly depends on the knot at hand. More precisely, $\alpha$ seems to depend only on the number of primary knots (factors) and increases by one for each added factor in the knot factorization.

In practice, all the characterizations of knots involve only their projection in a two-dimensional (2D) plane. Therefore, nothing prevents us from considering a twodimensional model for knotted polymers. Such a model should be in principle much simpler to study, both analytically and numerically. The aim of this paper is precisely to introduce and study numerically a particular model of "projected" polygons on a 2D lattice, describing 2D knotted polymers. In a 2D model for knotted polymers, the self-avoiding constraint in two dimensions is released to allow for what we call "crossings". By crossings, we actually mean two strands of the chain passing on top of each other in the projection. The object remains self-avoiding in the sense that the underlying and overlying strands cannot be exchanged by passing through each other. With this restriction, each closed polymer has a fixed knot type, which can be preserved in the deformations. Still the object is two-dimensional in the sense that its entropy corresponds to $2 \mathrm{D}$ deformations only. In particular, we will recover in some limits some of the usual exponents of 2D self-avoiding walks (SAW).

The paper is organized as follows. In the first section, we introduce our lattice model of projected self-avoiding polygons. Section 2 describes the local elementary moves which we use to deform the polygon and explore its accessible phase space at a fixed knot topology. The issue of ergodicity is also discussed in this section where arguments for a proof of ergodicity within a fixed knot type (but not a full proof) are given. The elementary moves are then performed according to a Monte Carlo Metropolis algorithm, drawn to reproduce the most natural grand canonical statistical weight, with a fugacity $K$ per bond of polygon, and a fugacity $w$ per crossing. The updating rules are discussed in section 3. Several improvements of the algorithm are described in section 4 . They involve both non local deformations and 


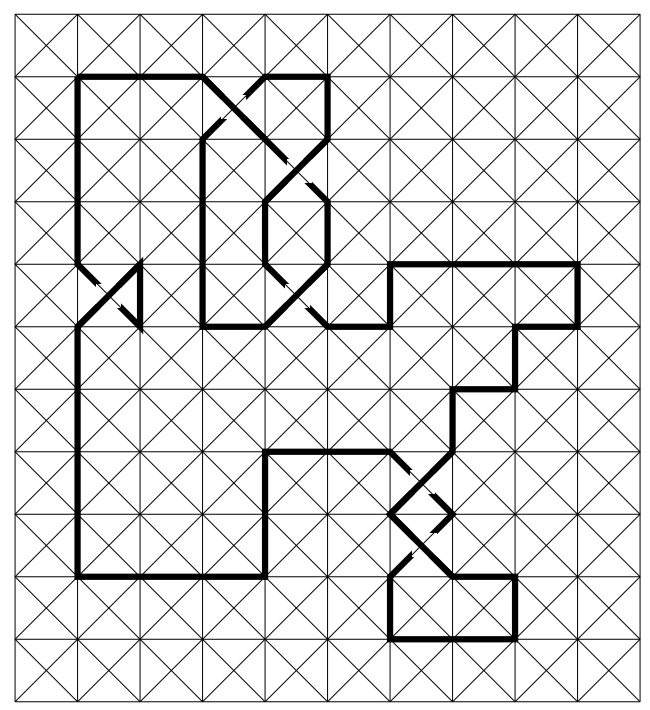

Figure 1: An example of a PSAP configuration on the square diagonal lattice with six crossings and the topology of a trefoil knot.

multiple chains run in parallel. Our numerical results are presented in sections 5, 6 and 7 for $w=1, w \rightarrow 0$ and $w>1$ respectively. We gather our conclusions in section 8.

\section{The Model}

We present here the model of Projected Self-Avoiding Polygons (PSAP's) that we shall use to describe two-dimensional knotted polymers. The model is defined on the square diagonal lattice in two dimensions, namely the simple two dimensional square lattice $Z^{2}$ completed with the diagonals of the squares. We treat each diagonal as an elementary edge of the lattice, i.e. we consider that the intersection point of the two diagonals is not a vertex of the lattice. The bonds of the polymer can sit on all the edges of the lattice, either vertical, horizontal or diagonal, with at most one bond per lattice edge. Different rules apply for the edges and vertices of the simple square lattice on the one hand and for the diagonal edges on the other hand. We impose a strict self-avoidance at the vertices of the square lattice, i.e. we do not allow two parts of the polymer to either cross each other or even to touch each other at these vertices. We allow crossings to take place inside a square at the intersection point of its two diagonal edges. More precisely, we use the diagonal edges only for crossings, i.e. we impose that a diagonal edge can be occupied if and only if the perpendicular diagonal edge in the same square is also occupied, with the two bonds on these edges forming a crossing. For each pair of occupied diagonal edges inside a square, we distinguish between two different possible crossings according to which of the two bonds of polymer passes on top of the other. We can thus view crossings as a two dimensional projection of a pair of bonds in three dimensions, with one bond 


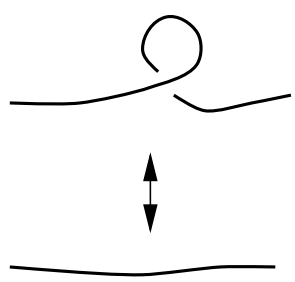

I

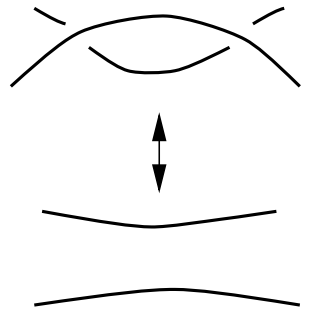

II

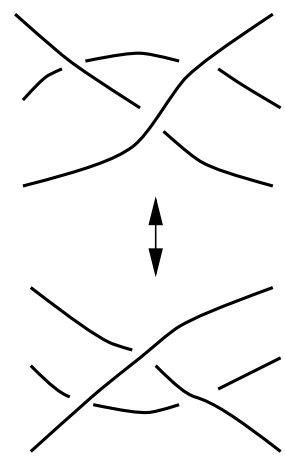

III

Figure 2: The three types (I, II and III) of Reidemeister moves on the projections of knots. Reversing all the crossings also corresponds to allowed transformations.

lying on top of the other. Finally, we limit ourselves to closed polymers. Figure 1 shows an example of an allowed configuration with six crossings and the topology of a trefoil knot.

Our model can be seen as a simple extension of the usual model describing selfavoiding polymer loops on the square lattice, also refered to as Self-Avoiding Polygons (SAP). The new ingredient here is the possibility for the polymer, which is strictly self-avoiding on the square lattice, to have crossings taking place on pairs of perpendicular diagonal edges inside a square. Again, these "crossings" are viewed as the two dimensional projection of two bonds of polymer passing on top of each other, with thus two distinct allowed configurations according to which of the two bonds is on top of the other. Thanks to this distinction, our model still describes a particular self-avoiding object in the sense that it mimics the projection in two dimensions of a polymer which would be self-avoiding in three dimensions. We will refer to our model as a model of Projected Self-Avoiding Polygons (PSAP's). Since we use closed polygons, a PSAP will in general form knots, i.e. will be the two-dimensional projection of a three dimensional knot. In this sense, we can speak of our model as describing two-dimensional knotted polymers.

To fully specify the model and to study the corresponding statistics, we need to assign to each PSAP configuration its weight. Since our aim is the study of knotted polymers of a fixed knot type, we want to attach a non-zero weight only to those configurations which have the desired knot-topology. We will be mainly interested below in rather simple topologies, i.e. that of the unknot $(\emptyset)$, that of the trefoil knot $\left(3_{1}\right)$, that of the figure eight knot $\left(4_{1}\right)$ and that of the composite knots made of two trefoils $\left(3_{1} \# 3_{1}\right)$ or a trefoil and a figure eight $\left(3_{1} \# 4_{1}\right)$. In practice, we will start with an initial configuration fixing the knot type and we will explore the phase space accessible by performing successive transformations (moves) on the PSAP which preserve its topology. As well known from knot theory, local deformations exist on the projection of a knot which preserve its topology. These deformations are classified 
as the Reidemeister moves [12], which are of three types as described in figure 2 . These topological deformations are sufficient to pass from any two configurations of the same knot type. The set of allowed moves in our lattice model will be described in detail in the next section. For all the accessible conformations of PSAP of a given knot type, we moreover weigh the configuration by a factor

$$
\pi(\mathcal{P})=\frac{n^{Q} K^{n} w^{c}}{G_{\tau}(Q, K, w)}
$$

where $n$ is the number of bonds of the PSAP configuration $\mathcal{P}$, with a fugacity $K$ per bond, and $c$ is the number of crossings, with a fugacity $w$ per crossing. We also artificially introduce a factor $n^{Q}$ with a factor $Q>1$ for numerical convenience. This factor is unimportant for averages at fixed $n$ (canonical ensemble) but it can improve the statistics in a grand-canonical ensemble with varying $n$. We typically take $Q=2$ in the following. The denominator in eq.(1) is the grand-canonical partition function at fixed knot-topology $\tau$, which normalizes the weights so that the total weight for all accessible configurations is equal to unity. For the knot-topology preserving moves, the above weight will dictate the probability to accept or reject the deformation.

\section{Local Elementary Moves}

In this section, we describe the local part of our algorithm, which is a grand-canonical implementation, since it involves changes in the number of bonds of the projected polygon. Our algorithm uses local elementary moves of four different types depending on how many elementary squares of $Z^{2}$ (plaquettes) are involved in the move. We will use single, double, 3-plaquette (or corner) and 6-plaquette moves. We first give here a description of all these elementary moves. The way we decide in the algorithm which type of move we attempt and with which probability we accept or reject the attempted move is described in the next section.

Single Moves Single Moves are performed on a single plaquette, and may involve length changes through the addition or subtraction of bonds in the PSAP. This moves are defined as follows: given a horizontal or vertical bond $\left(p_{i}, p_{i+1}\right)$ of the current PSAP, we pick a unit vector $e_{i}$ perpendicular to $\left(p_{i}, p_{i+1}\right)$. This defines a plaquette $\left(p_{i}, p_{i+1}, p_{i+1}+e_{i}, p_{i}+e_{i}\right)$. A move can occur only if the edge $\left(p_{i}+\right.$ $\left.e_{i}, p_{i+1}+e_{i}\right)$ is not occupied. The move depends on how many edges are occupied on the plaquette. If exactly two edges are occupied, we exchange occupied and unoccupied edges. This corresponds to a on bead flip (transformation $S_{I}$ in figure [3). If only one edge is occupied, we shift $\left(p_{i}, p_{i+1}\right)$ one lattice spacing in the direction of $e_{i}$ and complete the PSAP by two additional bonds, either on the sides, creating a kink (transformation $S_{I I}$ in figure 3), or on the diagonals, creating a crossing (transformation $S_{I V}$ or $S_{I V}^{\prime}$ in figure (i). If exactly three edges are occupied, we move $\left(p_{i}, p_{i+1}\right)$ to $\left(p_{i}+e_{i}, p_{i+1}+e_{i}\right)$ and delete the two other bonds. The result is either a kink deletion (transformation $S_{I I I}$ in figure 31) or a crossing deletion (transformation $S_{V}$ or $S_{V}^{\prime}$ in figure (4). 

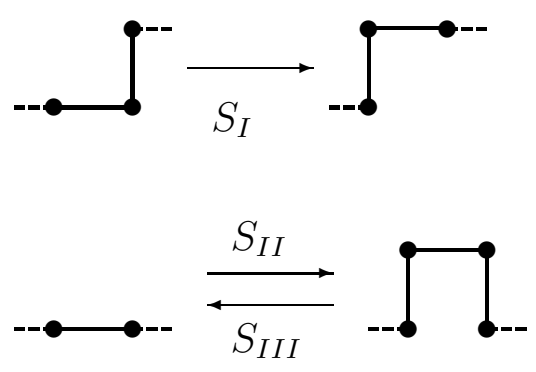

Figure 3: Single local $c$-preserving moves. $\left(S_{I}\right)$ One-bead flip $(\Delta n=0)$. $\left(S_{I I}\right)$ Kink insertion $(\Delta n=+2)$. $\left(S_{I I I}\right)$ Kink deletion $(\Delta n=-2)$.

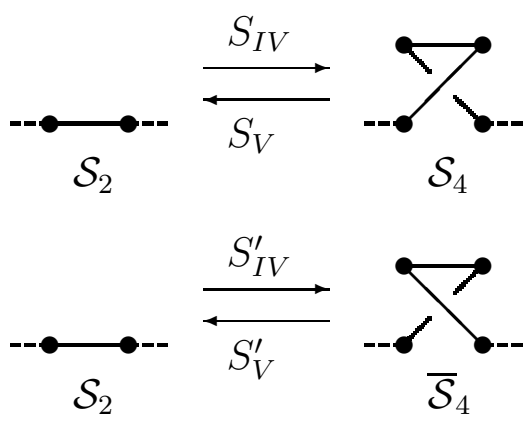

Figure 4: Single local $c$-changing moves: Reidemeister I moves. $\left(S_{I V}, S_{I V}^{\prime}\right)$ Crossing insertion $(\Delta c=+1)$. $\left(S_{V}, S_{V}^{\prime}\right)$ Crossing deletion $(\Delta c=-1)$.

The single moves can be classified into two different sub-groups depending on whether they do or do not preserve the number of crossings $c$ of the PSAP. The $c$-preserving moves illustrated in figure 3 are known in the literature as the BFACF moves [13, 14, 15]. While the move $S_{I}$ (one-bead flip) is $n$-preserving, the moves $S_{I I}$ (kink insertion) and $S_{I I I}$ (kink deletion) change the length of the PSAP respectively by $\Delta n=+2$ and $\Delta n=-2$. The $c$-changing moves are illustrated in figure 1 . In this case, in addition to the change $\Delta n= \pm 2$ in the length of the PSAP, the number of crossings changes by an amount $\Delta c=+1$ (moves $S_{I V}$ and $S_{I V}^{\prime}$ ) or $\Delta c=-1$ (moves $S_{V}$ and $S_{V}^{\prime}$ ). The moves $S_{I V}^{\prime}$ and $S_{V}^{\prime}$ are identical to the moves $S_{I V}$ and $S_{V}$ apart from the reversing of the crossing between $\overline{\mathcal{S}}_{4}$ and $\mathcal{S}_{4}$. All these $c$-changing moves correspond to a Reidemeister I transformation.

Double Moves A double move is performed on two adjacent plaquettes. Double moves are selected by first choosing, along the current PSAP, a vertex $p_{i}$. Different kinds of double moves are considered, depending on the relative orientations of the two bonds $\left(p_{i-1}, p_{i}\right)$ and $\left(p_{i}, p_{i+1}\right)$ shared by the vertex $p_{i}$ (see figure 5): 


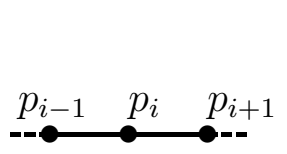

(a)

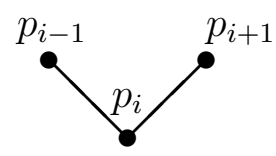

(b)

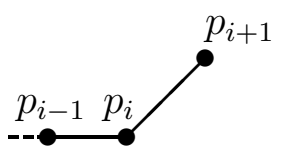

(c)

Figure 5: Different cases considered for double moves: (a) parallel vertical or horizontal bonds; (b) perpendicular diagonal bonds; (c) bonds at $135^{\circ}$.

Case of parallel vertical or horizontal bonds: If the two bonds are parallel, we choose one of the two possible unit vectors $e_{i}$ perpendicular to $\left(p_{i-1}, p_{i+1}\right)$, and we check if the vertex $p_{j}=p_{i}+e_{i}$ belongs to the current PSAP configuration. If it does, we look for a 2-plaquette configuration $\mathcal{D}_{1}$ such as that depicted in figure 6. If this 2-plaquette configuration is encountered, we make the local transformation $D_{I}$ by exchanging $p_{i}$ and $p_{j}=p_{i}+e_{i}$, leading to the configuration $\mathcal{D}_{2}$ with two more crossings, or make the similar transformation $D_{I}^{\prime}$ leading to the reversed configuration $\overline{\mathcal{D}}_{2}$.

Case of perpendicular diagonal bonds: If $\left(p_{i-1}, p_{i}\right)$ and $\left(p_{i}, p_{i+1}\right)$ are on perpendicular diagonal edges, the PSAP necessarily has two crossings involving two strands of polygon. We check that we have a configuration of type $\mathcal{D}_{2}$ or $\overline{\mathcal{D}}_{2}$ i.e. that the two strands are not entangled (see figure 6). If so, we then make the local transformation $D_{I I}$ or $D_{I I}^{\prime}$ to suppress the two crossings.

Case of bonds at $135^{\circ}$ : If one of the bonds, say $\left(p_{i-1}, p_{i}\right)$ is vertical or horizontal, and the other bond $\left(p_{i}, p_{i+1}\right)$ is at $135^{\circ}$ on a diagonal edge, the procedure is analogous to the case of parallel bonds, with $e_{i}$ the unit vector perpendicular to $\left(p_{i-1}, p_{i}\right)$ and inside the convex sector $\left(p_{i-1}, p_{i}, p_{i+1}\right)$. After checking that the vertex $p_{j}=p_{i}+e_{i}$ belongs to the current PSAP, we look for a 2-plaquette configuration such as the one depicted in figure 7 . We then let the crossing diffuse one step, according to transformation $D_{I I I}$ of figure 7. A similar procedure is used for the configuration $\overline{\mathcal{D}}_{3}$ obtained by reversing the crossing of $\mathcal{D}_{3}$.

Finally, no double move is performed if the two consecutive bonds shared by $p_{i}$ belong to the same plaquette. In this case a corner move (see below) will be attempted instead.

Transformations $D_{I}$ and $D_{I I}$ can be though of as Reidemeister II moves while transformation $D_{I I I}$ is simply a diffusion of the crossing to a neighboring pla- 


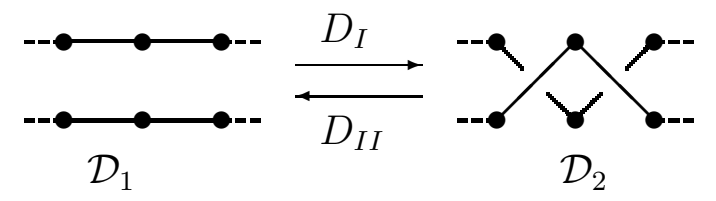

Figure 6: Double $c$-changing moves : Reidemeister II moves.

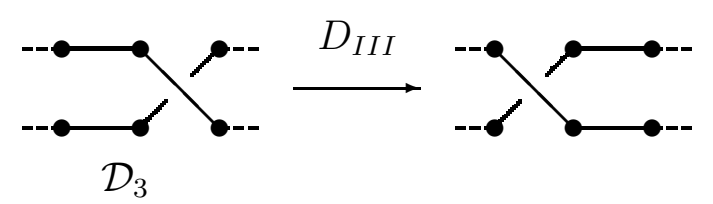

Figure 7: Double $c$-preserving move: diffusion of a crossing.

quette.

In addition to the configurations described above, there are also 2-plaquette configurations in which the two involved strands of the PSAP are consecutive. We call these configurations degenerate since can be transformed one into another by the same set of double moves above. Some of such configurations are illustrated in figure 8 .

Corner Moves . In these moves, three plaquettes of the underlying squared lattice are involved. As for the double moves, we first choose a vertex $p_{i}$ of the current PSAP. A corner move can be performed if the bonds $\left(p_{i-1}, p_{i}\right)$ and $\left(p_{i}, p_{i+1}\right)$, shared by the chosen vertex $p_{i}$, are on the two consecutive edges of a square, as illustrated in figure 9. In this case, we check if the vertex $p_{j}=-p_{i}+$ $p_{i+1}+p_{i-1}$. belongs to the current PSAP. If so, we look for local configurations involving three plaquettes such as those depicted in figure $10\left(\mathcal{C}_{1}, \mathcal{C}_{2}\right)$ and in figure $11\left(\mathcal{C}_{3}\right)$. If the local 3 -plaquette configuration does not correspond to any of such configurations no move is performed. Otherwise, we attempt one of the corner moves illustrated in figures 10 and 11. We can think of the corner

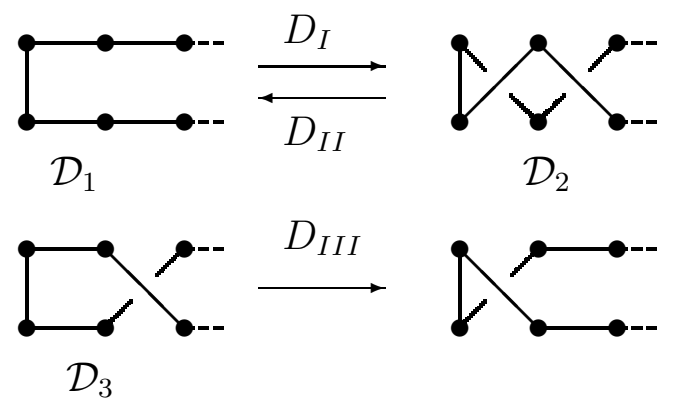

Figure 8: Degenerate configurations for double moves. 


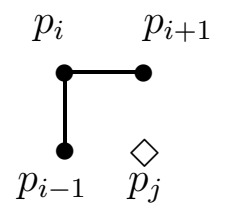

Figure 9: Starting configuration for a corner move.

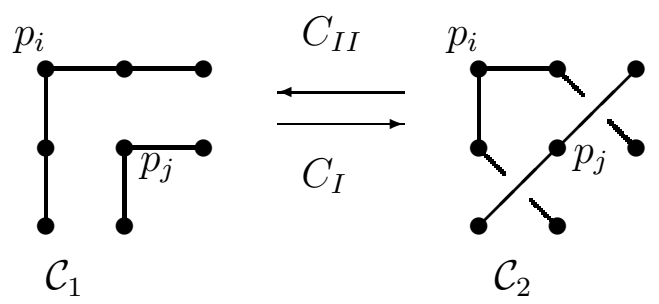

Figure 10: Corner $c$-changing move: Reidemeister II move around a corner.

moves $C_{I}$ and $C_{I I}$ as Reidemeister II moves, whereas the corner move of figure 11 corresponds to a diffusion of a crossing through the corner. Note that this diffusion around a corner also involves a rotation of the the crossing on the square lattice. Corner moves $C_{I}^{\prime}$ and $C_{I I}^{\prime}$ connecting configuration $\mathcal{C}_{1}$ to $\overline{\mathcal{C}}_{2}$ are also possible. In addition, as for the double moves, there are again degenerate configurations that can be transformed one into another by the same corner moves described above. Some of them are illustrated in figure 12 .

6-plaquette Moves (Reidemeister III moves). This moves are performed by choosing a diagonal bond in the current PSAP. This bond singles out a crossing. We then look for the presence of two neighboring crossings along two adjacent diagonal directions, chosen at random. We finally look for configurations such as the one depicted in figure 13. The performed move $R_{I}$ corresponds to a Reidemeister III move. Here again degenerate configurations exist which can be changed one into another.

Note that double, corner and 6-plaquette moves do not change the number $n$ of bonds in the PSAP.

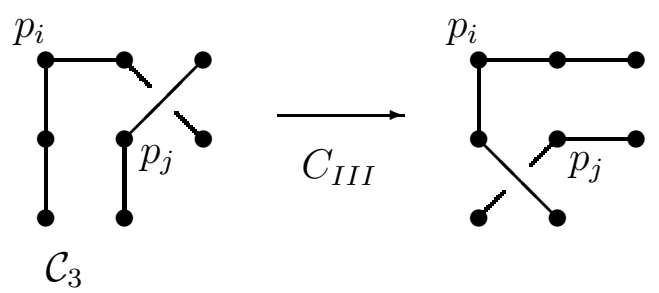

Figure 11: Corner $c$-preserving move : diffusion of a crossing around a corner. 


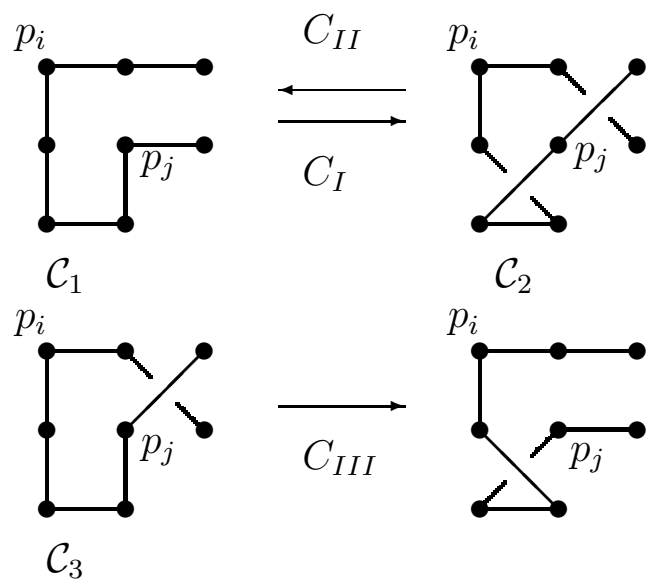

Figure 12: Degenerate cases of corner moves.

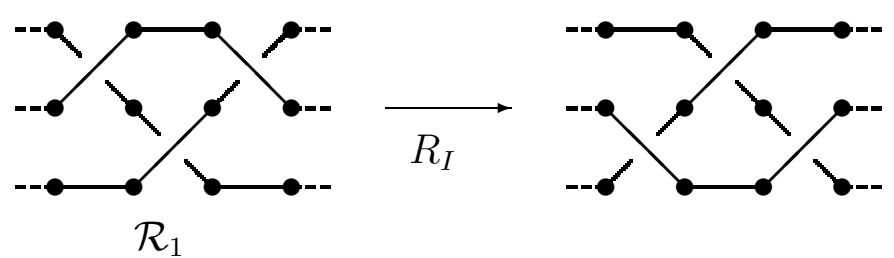

Figure 13: 6-plaquette $c$-preserving move : Reidemeister III move.

To end this section let us mention that:

- For all the moves drawn above, equivalent moves exist which are obtained by $90^{\circ}$ rotations or mirror reflections.

- If after our checks we do not find one of the above described environments, no move is performed.

- As described in the next section we have an exhaustive procedure to decide which type of move will be attempted.

All the moves above clearly preserve the knot-topology. Therefore any possible deformation will maintain the PSAP inside the set of conformations having the same knot type as the conformation one started with. In the following, we will assume that the algorithm is ergodic, although a full proof of ergodicity goes far beyond our goal. By ergodicity, we mean that starting from a given conformation, any other conformation with the same knot-topology can be obtained by a series of successive elementary moves. In the absence of crossings, our algorithm reduces to the BFACF algorithm [13, 14, 15, which is known to be ergodic. More precisely, the BFACF moves (moves $S_{I}, S_{I I}$ and $S_{I I I}$ ) allow to deform any configuration of a set of selfavoiding paths onto any other configuration with the same topology and, if some of the paths are open, with the same positions of the end-points. Our algorithm completes the BFACF algorithm with knot-topology preserving moves allowing the 
three types of Reidemeister moves, as well as the migration of a crossing along the PSAP.

Although we have no rigorous proof of ergodicity for our algorithm, let us present here what could be the sketch of a proof. Before we proceed, let us note that we can limit ourselves without loss of generality to conformations on which none of the transformations $S_{V}, C_{I I}$ or $D_{I I}$ (which decrease the number of crossings) can be performed. If such a transformation can be performed, then we do perform it (possibly recursively) so as to eliminate the spurious crossings. We are thus left with configurations where the suppression of a crossing requires more than one elementary move. We then divide the set of remaining conformations with a fixed knot type into subsets of conformations which have precisely the same (topologically speaking) knot projection. The topology of such a subset is characterized by a fixed number of crossings, and a fixed prescription for the connectivity of these crossings. A proof of ergodicity can then be established in two steps:

- (1) Proving the ergodicity within a subset, i.e. for a fixed topology of the projection (i.e. a fixed number of crossings and a fixed set of connections between them).

- (2) Showing the possibility to pass from a particular element (representative) of one subset to a particular element of another subset if the two different projections represent the same knot.

Since two projections representing the same knot can be related by a series of Reidemeister moves, it is enough to prove point (2) that representatives of two different subsets differing by a Reidemeister move can be related one to the other. A good choice of representatives is what could be called expanded configurations, i.e. configurations in which all the crossings are well separated. In a grand-canonical ensemble, such configurations always exist, whatever big is the number of crossings in the projection. A Reidemeister move will involve at most three crossings in the projection. For well separated crossings, the figures 14, 15 and 16 show how to perform a Reidemeister transformation of type I, II or III respectively by use of our elementary moves. For a Reidemeister I move, we can use the BFACF moves to shrink a path with neighboring end-points onto the segment joining these end-points, then use the move $S_{I V}$ or $S_{V}$ to create or destroy a crossing, and use BFACF moves again to re-inflate the path. For Reidemeister moves II and III, the idea is to use the BFACF moves to deform the paths (with fixed end-points) connecting crossings so as to create channels along which the crossings can migrate. The migration of crossings along a channel is performed by use of the elementary moves $D_{I I I}$ or $C_{I I I}$ (at a corner) until the crossings come in contact and an elementary move $D_{I}$ or $D_{I I}$ (Reidemeister II), or $R_{I}$ (Reidemeister III) can be performed. The target expanded configuration can then be reached again by creating channels, moving the crossings to their final positions, and eventually re-deforming the connecting paths. Note that for all these deformations, the moves $C_{I}$ and $C_{I I}$ have not been used. This is because these moves are not independent and can be performed by a sequence of $D_{I}, D_{I I}, D_{I I I}, C_{I I I}$ and 


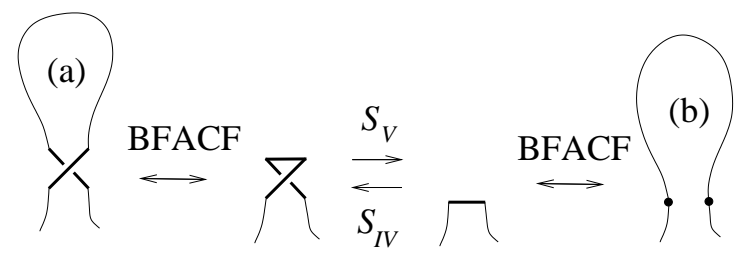

Figure 14: The passage between two expanded configurations (a) and (b) related by a Reidemeister I transformation. Thin lines indicate an arbitrary (supposedly drawn a the square lattice) path joining the crossings. For each step of the transformation, we indicate the type of elementary move involved.
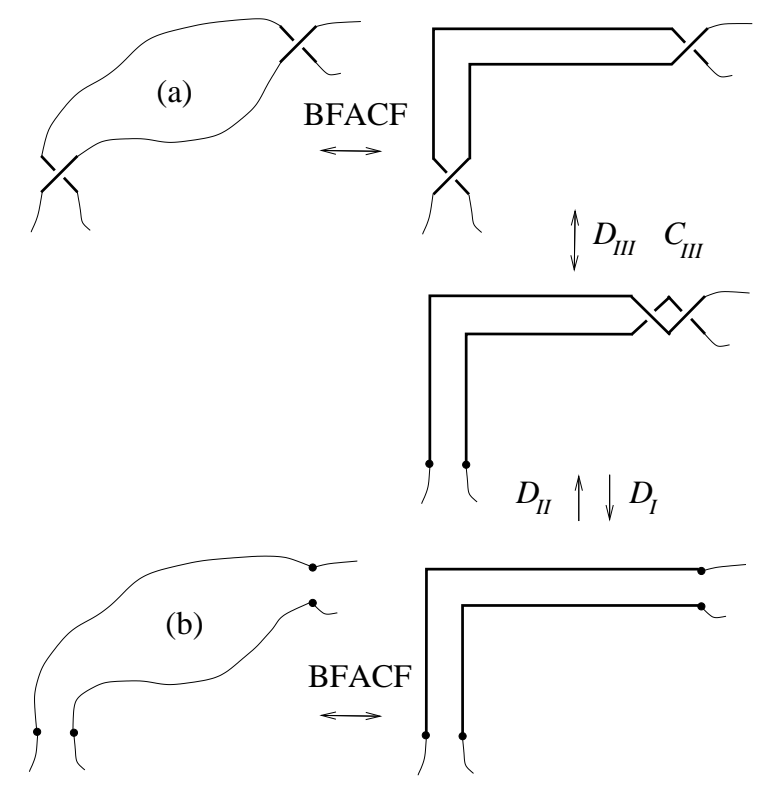

Figure 15: The passage between two expanded configurations (a) and (b) related by a Reidemeister II transformation.

BFACF moves. Still in a Monte Carlo program, it is worth implementing them to accelerate the algorithm.

Let us now come to the point (1) of our argument, i.e the ergodicity of the algorithm inside the subset of conformations having the same projection. A topological deformation will involve now only a displacement of the moves and a deformation of the paths connecting them, keeping the global connectivity structure. No Reidemeister move and in particular no creation of crossings have to be performed. Since BFACF moves allow to freely deform the already existing (i.e. with non-zero initial length) paths, and the moves $D_{I I I}$ and $C_{I I I}$ allow to move any crossing along a channel of connecting paths, the only possible source of blocking is due to situations where crossings have zero length connecting paths, i.e. are nearest neighbors of other 


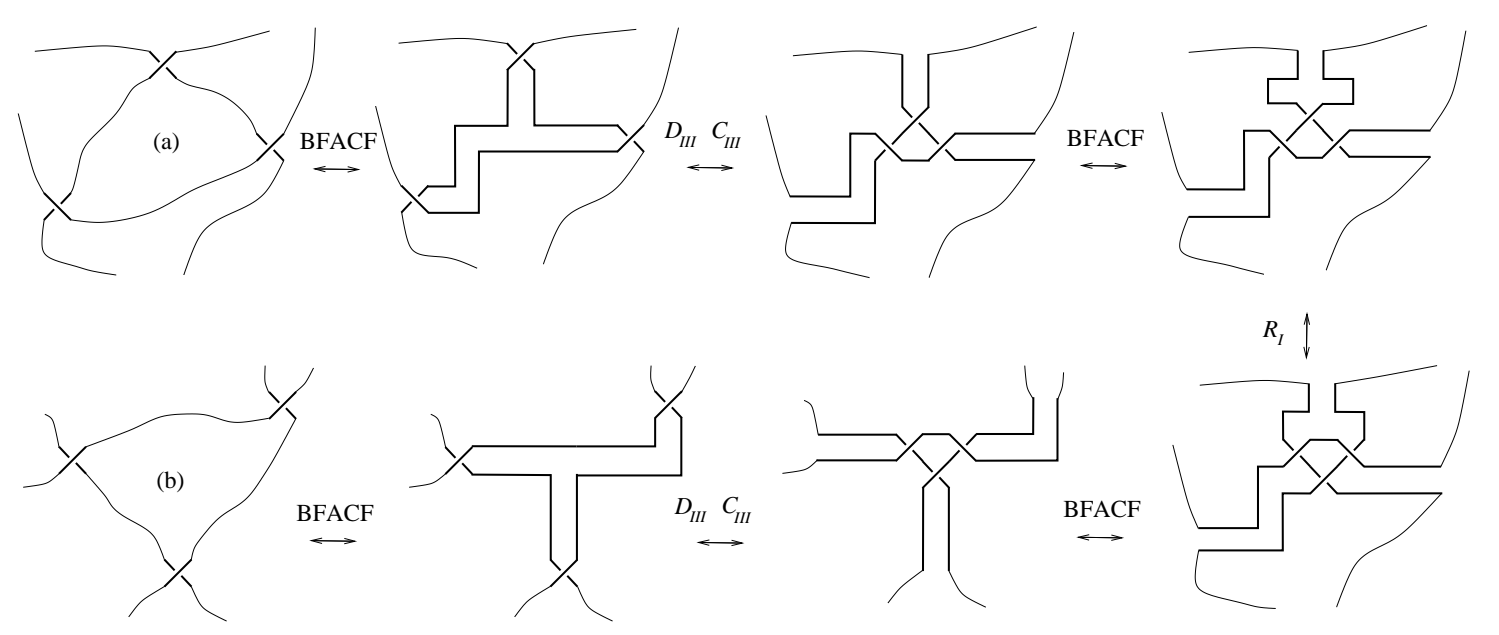

Figure 16: The passage between two expanded configurations (a) and (b) related by a Reidemeister III transformation.

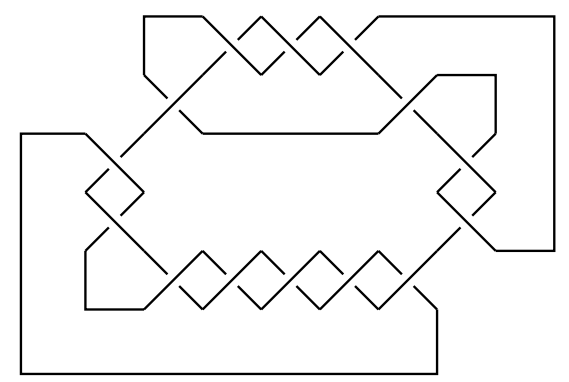

Figure 17: A blocked configuration with a loop of 14 alternating crossings.

crossings, without path between them. Indeed, our moves do not allow us to move several crossings at once. For this situation to lead to a real blocking, one moreover needs a set of neighboring crossings whose centers form a loop on the dual square lattice (the centers of the squares), so that each crossing in the loop in blocked on both sides (see an example in figure 17). Since spurious crossings have already been removed, these loop conformations require moreover many alternating crossings (at least eight) and correspond to entangled situations with a complex knot topology. We do not expect such conformations to belong to the simple knot topologies that we study here where we consider knots made only of trefoils and figure eight primary knots.

\section{$3 \quad$ Updating and Reversibility}

We will now discuss the updating of our system, i.e. how we decide which type of move to attempt and with which probability we accept the move. These choices are dictated by a condition of reversibility of the algorithm and by the nature of the 
invariant limiting distribution which we want to reach within an ergodicity class, i.e. the weight $\pi(\mathcal{P})$ of eq.(1) which we assign to a configuration $\mathcal{P}$ of the PSAP.

The implementation of the algorithm is "Metropolis style". Let $\mathcal{P}_{1}$ be the current PSAP with $n_{1}$ bonds and $c_{1}$ crossings. A tentative move of type $M$ is chosen according to a procedure which we describe below. It may or may not be possible. If it is, it produces a new configuration $\mathcal{P}_{2}$ with length $n_{2}$ and number of crossings $c_{2}$. Then we accept the move with the acceptance rate

$$
\operatorname{Acc}\left(\mathcal{P}_{1} \rightarrow \mathcal{P}_{2}\right)=A(M) \min \left[1, K^{n_{2}-n_{1}}\left(\frac{n_{2}}{n_{1}}\right)^{Q-1}\right] \min \left[1, w^{c_{2}-c_{1}}\right]
$$

The two $\min [\cdot]$ terms correspond to the usual Metropolis acceptance rate dictated by the limiting distribution of eq. (1). In particular, the term involving the ratio $n_{2} / n_{1}$ is necessary to reproduce the $n^{Q}$ dependence of $\pi(\mathcal{P})$. In addition, we also introduce a coefficient $A(M) \leq 1$ depending on the type of move $M$ attempted, and which we will determine later. The probability to pass from the configuration $\mathcal{P}_{1}$ to the configuration $\mathcal{P}_{2}$ is thus

$$
\operatorname{Prob}\left(\mathcal{P}_{1} \rightarrow \mathcal{P}_{2}\right)=A(M) \min \left[1, K^{n_{2}-n_{1}}\left(\frac{n_{2}}{n_{1}}\right)^{Q-1}\right] \min \left[1, w^{c_{2}-c_{1}}\right] \times B\left(\mathcal{P}_{1}, M\right)
$$

where $B\left(\mathcal{P}_{1}, M\right)$ is the probability to have selected the move $M$ among all the tentative moves which could have been tried on $\mathcal{P}_{1}$. To be sure that the Markov chain converges to the limiting distribution $\pi(\mathcal{P})$, we impose on $\operatorname{Prob}\left(\mathcal{P}_{1} \rightarrow \mathcal{P}_{2}\right)$ the detailed balance condition

$$
\pi\left(\mathcal{P}_{1}\right) \operatorname{Prob}\left\{\mathcal{P}_{1} \rightarrow \mathcal{P}_{2}\right\}=\pi\left(\mathcal{P}_{2}\right) \operatorname{Prob}\left\{\mathcal{P}_{2} \rightarrow \mathcal{P}_{1}\right\}
$$

This will dictate the choice of $A(M)$ below.

The implementation of the algorithm is as follows:

1. To attempt a local move on the current configuration $\mathcal{P}_{1}$ we first select with probability $q$ a bond at random on the configuration or with probability $(1-q)$ a vertex at random on the configuration. The number $q$ is a free parameter which we can adjust in our simulation.

2. Case of bond selection. In this case, after having chosen the bond at random among the $n_{1}$ bonds of $\mathcal{P}_{1}$, we attempt a 6 -plaquettes move if the bond is a diagonal bond, or a single move if the bond is vertical or horizontal.

(i) 6-plaquette move. Starting from the chosen diagonal bond, we look for a configuration such as those in figure 13 (or their degenerate companions). If such a configuration is not found the move is rejected. Otherwise, the outcome is a PSAP $\mathcal{P}_{2}$ with $n_{2}=n_{1}$ and $c_{2}=c_{1}$, and

$$
\operatorname{Prob}\left\{\mathcal{P}_{1} \rightarrow \mathcal{P}_{2}\right\}=A\left(R_{I}\right) B\left(\mathcal{P}_{1}, R_{I}\right) \quad ; \quad B\left(\mathcal{P}_{1}, R_{I}\right)=\frac{2 q}{n_{1}} \times \frac{1}{4}
$$


The factor $2 / n_{1}$ is the probability to have chosen among $n_{1}$ bonds one of the two bonds forming the crossing which is the starting point of the Reidemeister III move, and the factor $1 / 4$ is the probability to have selected the correct diagonal directions of the two other crossings involved. The important point is that every 6-plaquette move has a unique inverse (see figure 13) by design which is also a 6-plaquette move of the same type. Detailed balance holds by choosing

$$
A\left(R_{I}\right)=1
$$

since, by exchanging $\mathcal{P}_{1}$ and $\mathcal{P}_{2}$, and using $n_{2}=n_{1}$, we have $\operatorname{Prob}\left\{\mathcal{P}_{2} \rightarrow\right.$ $\left.\mathcal{P}_{1}\right\}=\operatorname{Prob}\left\{\mathcal{P}_{1} \rightarrow \mathcal{P}_{2}\right\}$ and $\pi\left(\mathcal{P}_{1}\right)=\pi\left(\mathcal{P}_{2}\right)$.

(ii) Single move. For the single moves, we proceed as described in the previous section. If the considered plaquette has two occupied edges, we do a bead flip $S_{I}$. Since the bead flip $S_{I}$ keeps the number of bonds and crossings unchanged, the detailed balance follows by noticing that any such move has its own inverse by design. We can thus take

$$
A\left(S_{I}\right)=1 .
$$

If the considered plaquette has a single occupied edge, we must choose between the three moves corresponding to an addition of two bonds $\left(S_{I I}, S_{I V}\right.$ and $\left.S_{I V}^{\prime}\right)$ with $n_{2}-n_{1}=2$ and $\Delta c=0$ or 1 . We choose at random one of these three moves with even probability $1 / 3$. For convenience, the acceptance rates $A\left(S_{I I}\right), A\left(S_{I V}\right)$ and $A\left(S_{I V}^{\prime}\right)$ will be taken all equal to $A_{+2}^{(S)}$. To fix them, we need to consider the reverse moves, which are performed on a plaquette which has three occupied edges. In this case, the current configuration dictates without ambiguity which transformation $S_{I I I}, S_{V}$ or $S_{V}^{\prime}$ can be done. Again we take $A\left(S_{I I I}\right), A\left(S_{V}\right)$ and $A\left(S_{V}^{\prime}\right)$ all equal to $A_{-2}^{(S)}$. If $\mathcal{P}_{1}$ and $\mathcal{P}_{2}$ are the two configurations mutually exchanged by some of these transformations, with $n_{2}=n_{1}+2$, we have

$$
\operatorname{Prob}\left\{\mathcal{P}_{1} \rightarrow \mathcal{P}_{2}\right\}=A_{+2}^{(S)} \frac{q}{n_{1}} \frac{1}{2} \frac{1}{3} \min \left[1, K^{n_{2}-n_{1}}\left(\frac{n_{2}}{n_{1}}\right)^{Q-1}\right] \min \left[1, w^{c_{2}-c_{1}}\right]
$$

while

$$
\operatorname{Prob}\left\{\mathcal{P}_{2} \rightarrow \mathcal{P}_{1}\right\}=A_{-2}^{(S)} \frac{q}{n_{2}} \frac{1}{2} \min \left[1, K^{n_{1}-n_{2}}\left(\frac{n_{1}}{n_{2}}\right)^{Q-1}\right] \min \left[1, w^{c_{1}-c_{2}}\right]
$$

Choosing

$$
A_{+2}^{(S)}=1 ; \quad A_{-2}^{(S)}=\frac{1}{3}
$$

the detailed balance

$$
n_{1}^{Q} K^{n 1} w^{c_{1}} \operatorname{Prob}\left\{\mathcal{P}_{1} \rightarrow \mathcal{P}_{2}\right\}=n_{2}^{Q} K^{n 2} w^{c_{2}} \operatorname{Prob}\left\{\mathcal{P}_{2} \rightarrow \mathcal{P}_{1}\right\}
$$

is then satisfied. 
3. Case of vertex selection. We now suppose that we have selected a vertex, which occurs with probability $(1-q)$. In this case, we attempt either a double or a corner move, depending on the relative position of the two bonds $\left(p_{i-1}, p_{i}\right)$ and $\left(p_{i}, p_{i+1}\right)$ shared by the chosen vertex. For the relative orientations of figure 5 , a double move is attempted; for the relative orientation of figure 9, a corner move is attempted. In both cases the number of bonds remains constant $(\Delta n=0)$. For the transformations $D_{I I I}$ and $C_{I I I}$ in which the number of crossings also remains unchanged (figure 7 and figure 11) the detailed balance holds clearly with

$$
A\left(D_{I I I}\right)=A\left(C_{I I I}\right)=1
$$

by noticing that any such move has its own inverse by design. For the remaining cases (figure 6 and figure 10) the number of crossings between $\mathcal{P}_{1}=\mathcal{D}_{1}$ (or respectively $\mathcal{C}_{1}$ ) and $\mathcal{P}_{2}=\mathcal{D}_{2}$ or $\overline{\mathcal{D}_{2}}$ (or respectively $\mathcal{C}_{2}$ or $\overline{\mathcal{C}_{2}}$ ) varies by an amount $c_{2}-c_{1}=2$ and we simply have to account for the fact that there are two ways to add the crossings while there is just one way to suppress them. Setting $A\left(D_{I}\right)=A\left(C_{I}\right)=A_{+2}^{(D, C)}$ and $2 A\left(D_{I I}\right)=A\left(C_{I I}\right)=A_{-2}^{(D, C)}$, we have

$$
\operatorname{Prob}\left\{\mathcal{P}_{1} \rightarrow \mathcal{P}_{2}\right\}=A_{+2}^{(D, C)} \times\left\{\begin{array}{l}
2 \\
1
\end{array}\right\} \times \frac{(1-q)}{n_{1}} \times\left\{\begin{array}{c}
\frac{1}{2} \\
1
\end{array}\right\} \times \frac{1}{2} \times \min \left[1, w^{2}\right]
$$

where the first line is for a double move and the second line for a corner move. The extra coefficients 2 and $1 / 2$ in the case of a double move come respectively from the two different vertices which select the pair of parallel strands under consideration and from the probability $1 / 2$ to make the correct choice of direction $e_{i}$ in which we move the vertex $p_{i}$. We have conversely

$$
\operatorname{Prob}\left\{\mathcal{P}_{2} \rightarrow \mathcal{P}_{1}\right\}=\left\{\begin{array}{l}
\frac{A_{-2}^{(D, C)}}{A_{-2}^{(D, C)}} \\
A_{-2}
\end{array} \times\left\{\begin{array}{l}
2 \\
1
\end{array}\right\} \times \frac{(1-q)}{n_{2}} \times \min \left[1, w^{-2}\right]\right.
$$

The detailed balance

$$
n_{1}^{Q} K^{n 1} w^{c_{1}} \operatorname{Prob}\left\{\mathcal{P}_{1} \rightarrow \mathcal{P}_{2}\right\}=n_{2}^{Q} K^{n 2} w^{c_{2}} \operatorname{Prob}\left\{\mathcal{P}_{2} \rightarrow \mathcal{P}_{1}\right\}
$$

is then satisfied by choosing

$$
A_{+2}^{(D, C)}=1 ; \quad A_{-2}^{(D, C)}=\frac{1}{2} .
$$

The analysis above may seem a little cumbersome. In practice, the only subtle point is the determination of the coefficients $A(M)$, the list of which is displayed in Table 1. These coefficients simply correct the fact that some transformations are in competition (like a kink creation and a crossing creation) while the reverse transformation has no competitor. As usual, the Metropolis criterion simply creates the correct "energy" factor. With the above choices for the acceptance coefficients $A(M)$, the transitions probabilities verify the detailed balance condition (雨) for all PSAP's $\mathcal{P}_{1}, \mathcal{P}_{2}$, with the invariant limit distribution of eq.(1) 


\begin{tabular}{||r|r||r|r||r|r||r|r||}
\hline \multicolumn{2}{||c||}{ Single Moves } & \multicolumn{2}{c||}{ Double Moves } & \multicolumn{2}{|||}{ Corner Moves } & \multicolumn{2}{||}{ 6-plaquette Moves } \\
\hline$S_{I}$ & 1 & $D_{I}$ & 1 & $C_{I}$ & 1 & $R_{I}$ & \\
$S_{I I}$ & 1 & $D_{I I}$ & $1 / 4$ & $C_{I I}$ & $1 / 2$ & & \\
$S_{I I I}$ & $1 / 3$ & $D_{I I I}$ & 1 & $C_{I I I}$ & 1 & & \\
$S_{I V}, S_{I V}^{\prime}$ & 1 & & & & & & \\
$S_{V}, S_{V}^{\prime}$ & $1 / 3$ & & & & & & \\
\hline
\end{tabular}

Table 1: Coefficients $A(M)$ for the different local moves.

\section{Improvements of the algorithm}

\subsection{Non-local Moves}

Algorithms based on local moves are known to have very long autocorrelation times which in turn lead to large statistical errors. For instance, it is known that, for the BFACF algorithm (which in our language simply corresponds to forbid crossings), the so called "exponential" autocorrelation time, which controls the relaxation of the Markov chain from an initial configuration to equilibrium, is infinite [16]. For the same algorithm, the so called "integrated" autocorrelation time, which controls the statistical error in measured quantities, is usually very large.

To remedy this problem, algorithms have been proposed [6, 17] which combine local moves and non-local moves. The non-local moves correspond to select two points far apart on the chain and to reverse the part of the chain between them. In these hybrid algorithms, the non-local moves hopefully ensure the rapid equilibration within subspaces of fixed length $n$, while local moves ensure equilibration between different values of $n$. Now if one wants to study the statistics of polygons with a fixed knot type, one must make sure that the non-local moves preserve the topology of the polygon. For the usual non-local moves performed on 3-dimensional Self-Avoiding Polygons in $Z^{3}$, this is in general not the case. One way to deal with this problem is then to check potential changes in the knot type by calculating a topological invariant such as the Alexander polynomial at every successful non-local move [6]. This is however computationally costly and it also has the disadvantage that the Alexander polynomial is not a perfect characterization of the knot type and distinct knots can have the same Alexander polynomial.

The situation is different in our model where the two-dimensional character of the PSAP allows the introduction of non-local moves that do preserve the topology of the PSAP. The non-local move we consider are performed by first selecting two vertices $\left(p_{1}\right.$ and $\left.p_{2}\right)$ in the PSAP with uniform probability. If these vertices are neighbors, no move is attempted; if not, the vertices separate the PSAP into two arcs, say $a_{1}$ and $a_{2}$. If the two arcs have no crossings in common we can perform a rotation by $180^{\circ}$ of the shortest one, say $a_{1}$ (see figure 18). Let $a_{1}^{\prime}$ be the image of $a_{1}$ under this rotation. If $a_{1}^{\prime}$ and $a_{2}$ do not intersect each other, no new crossing has been generated and the move is accepted. The important feature of this move is that it does not change the 

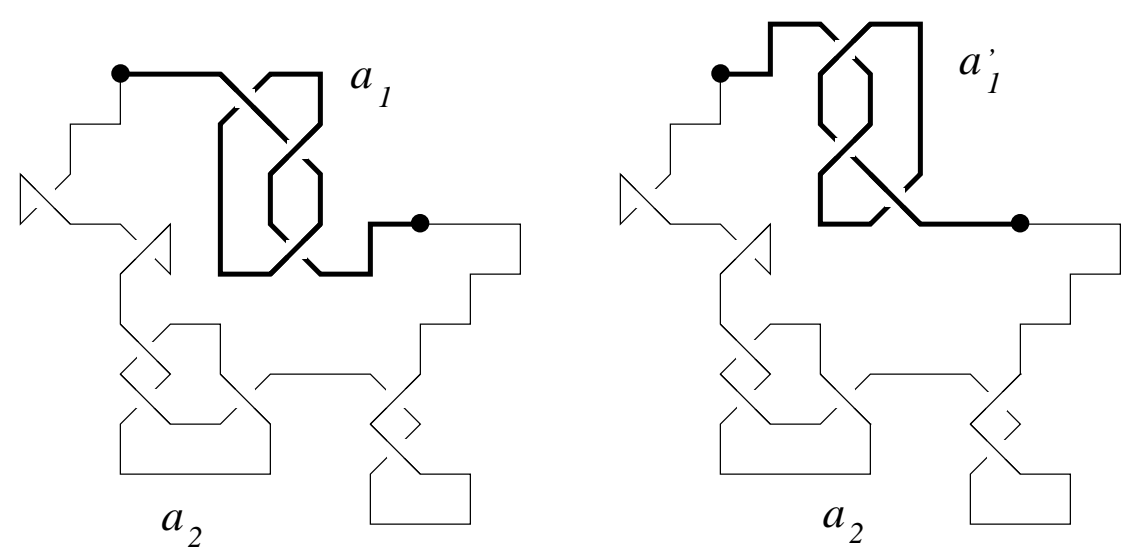

Figure 18: Non-local transformation of a PSAP. The arc $a_{1}^{\prime}$ is the image of $a_{1}$ by a rotation of $180^{\circ}$. The arc $a_{2}$ is unchanged.

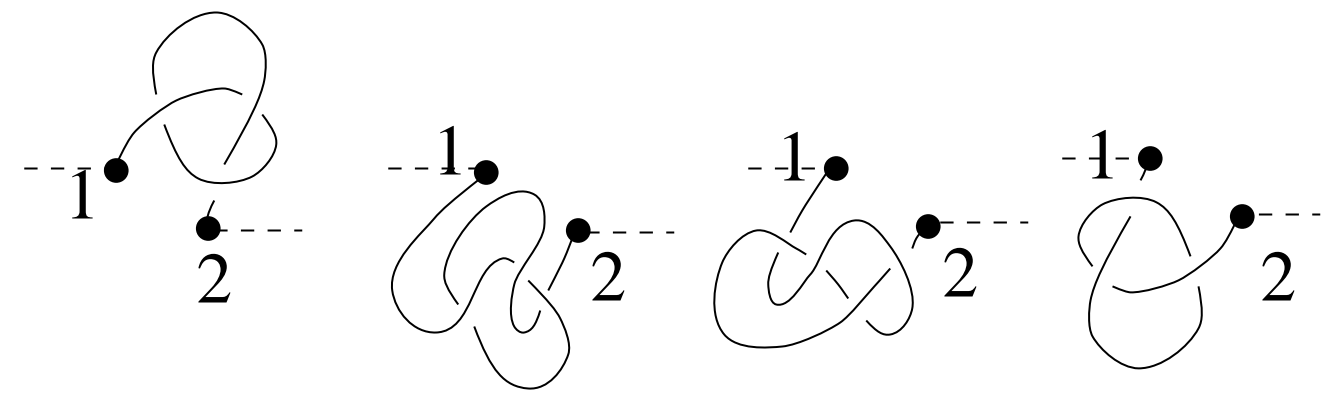

Figure 19: The passage from a trefoil to its image by a rotation of $180^{\circ}$ by use if local Reidemeister moves only. We indicate only the major intermediate steps.

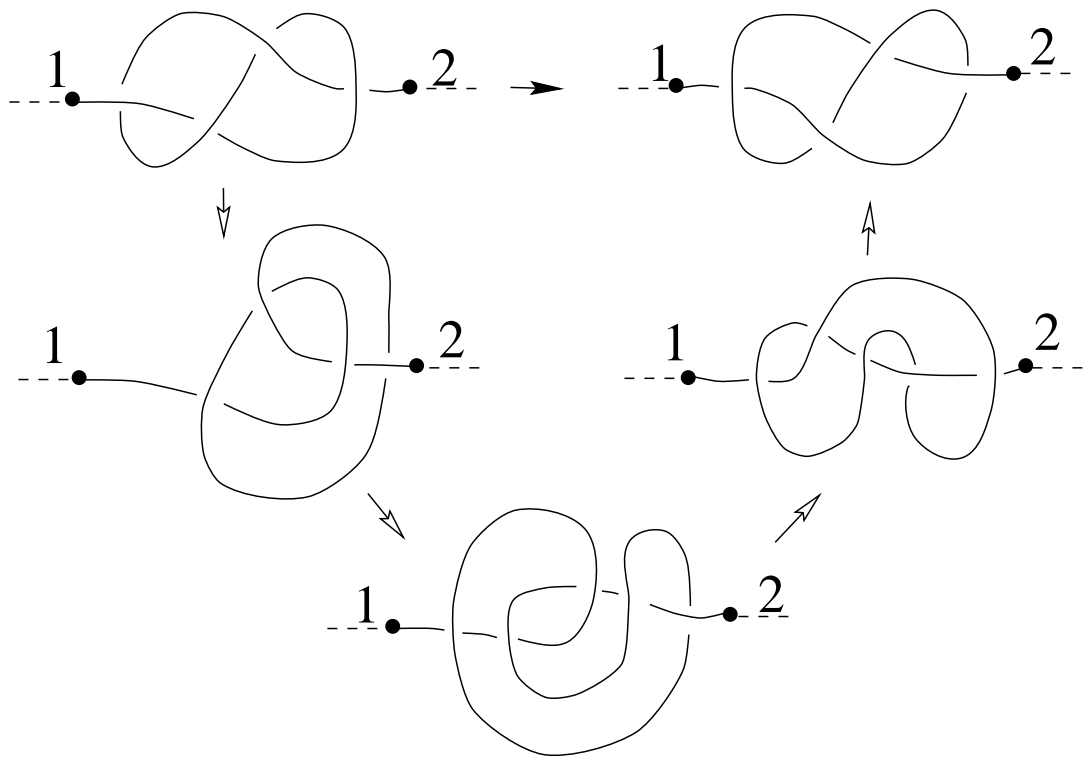

Figure 20: The passage from a figure eight to its image by a rotation of $180^{\circ}$ by use if local Reidemeister moves only. We indicate only the major intermediate steps. 
topology of the arc $a_{1}$ and therefore, since we avoid topological interaction between the arcs, it preserves the topology of the entire PSAP. To be more precise, the absence of crossings between the two arcs guarantees that the knots in $a_{1}$ and those in $a_{2}$ are well separated and the total knot can be viewed as the compositions of the two knots. It is known that the composition of two knots can give at most two different results according to whether their (arbitrarily fixed) orientations do or do not match in the composition. Note that our transformation reverses this matching of orientations. The result is however unique as soon as at least one of the knots is reversible (i.e. its two opposite orientations are in fact equivalent). This is the case for all the knots that we will use, which are made of trefoils $\left(3_{1}\right)$ and figure eight $\left(4_{1}\right)$ knots only, both of which are reversible. In practice, we can also argue that, for a trefoil $\left(3_{1}\right)$ or figure eight $\left(4_{1}\right)$ knot, the passage from the arc $a_{1}$ to its image $a_{1}^{\prime}$ can be done by using Reidemeister moves which do not involve the arc $a_{2}$. This is illustrated for the trefoil knot in figure 19 and for the figure eight knot in figure 20.

The non-local move described above is the simplest one that can be implemented. More sophisticated moves can be considered in which the constraint of having no crossings between $a_{1}$ and $a_{2}$ can be relaxed to weaker conditions. We however limited ourself to the simplest non-local moves without crossings.

In our algorithm, we therefore have an additional free parameter $0 \leq p<1$ which is the probability that we attempt non-local move rather than a local move.

\subsection{Multiple Markov Chain Method}

Another way to improve the slow convergence of the local algorithm is by sampling along a set of several Markov chains run in parallel.

This method, refered to as the Multiple Markov Chain (MMC) method, has been introduced by Geyer [18 and then adapted to interacting self-avoiding walks with fixed length by Tesi et al [19 (see also [20] for a more detailed analysis of the method).

We describe here a variant of this approach suitable for simulations in the grand canonical ( $n$ varying) ensemble. The starting point is the single Markov chain whose limiting distribution $\pi(\mathcal{P})$ depends on the step and crossing fugacities $K$ and $w$. Here we will consider a procedure with a fixed value of $w$, hence the $w$ dependence will be omitted in this section. For values of $K$ close to (and below) the critical value $K_{c}$ where the average length of the PSAP diverges, the Markov chain samples configurations with very large values of $n$ and the convergence of the algorithm is therefore very slow. On the contrary for a value $K^{\prime}$ of the step fugacity such that $K^{\prime}<<K_{c}$, the sampled configurations have small values of $n$ and the convergence of the local algorithm is fast. The idea is to select a set of values $K^{\prime}=K_{1}<K_{2}<$ $K_{3}<\ldots<K_{m}=K$ to interpolate between $K^{\prime}$ and $K$, so that $K_{j}$ and $K_{j+1}$ are close enough to ensure that there is a considerable overlap between the distribution at $K_{j}$ and that at $K_{j+1}$.

The $m$ Markov chains at $K_{1}, K_{2}, \ldots, K_{m}$ are evolved in parallel, and we let the chains interact by possibly exchanging conformations as follows: select at random two neighboring chains with fugacities $K_{j}$ and $K_{j+1}$. A trial move is an attempt to swap the two current conformations of these chains. If we denote by $\pi_{j}(\mathcal{P})$ the 


\begin{tabular}{|r||r|r||r|r||r|r||r|r||}
\hline \multicolumn{1}{|c||}{$K$} & \multicolumn{2}{c||}{ (1) local } & \multicolumn{2}{c||}{ (2) local + non-local } & \multicolumn{2}{c||}{ (3) local + MMC } & \multicolumn{2}{c|}{$(4)$ all } \\
\cline { 2 - 8 } & $\langle n\rangle$ & $\tau(n)$ & $\langle n\rangle$ & $\tau(n)$ & $\langle n\rangle$ & $\tau(n)$ & $\langle n\rangle$ & $\tau(n)$ \\
\hline 0.3760 & $76 \pm 4$ & $18 \pm 3$ & $75 \pm 3$ & $12 \pm 2$ & $75 \pm 1$ & $2.2 \pm 0.2$ & $75 \pm 1$ & $1.5 \pm 0.2$ \\
0.3771 & $110 \pm 11$ & $50 \pm 10$ & $100 \pm 6$ & $20 \pm 5$ & $111 \pm 4$ & $5.6 \pm 0.9$ & $112 \pm 2$ & $3.0 \pm 0.3$ \\
0.3780 & $160 \pm 19$ & $67 \pm 11$ & $188 \pm 18$ & $40 \pm 15$ & $188 \pm 9$ & $14 \pm 3$ & $192 \pm 9$ & $13 \pm 3$ \\
0.3783 & & & $260 \pm 35$ & $90 \pm 25$ & $231 \pm 17$ & $47 \pm 15$ & $259 \pm 19$ & $35 \pm 10$ \\
\hline
\end{tabular}

Table 2: Integrated autocorrelation times for the average length $\langle n\rangle$ of the unknot at $w=1$, for (1) the purely local algorithm, (2) the local + non-local algorithm, (3) the local + MMC algorithm and (4) local + non-local + MMC algorithm. Each run has a total of $10^{8}$ local moves and the unit of sampling is $10^{4}$ local moves. The data which are not reliable are omitted.

equilibrium weight of the state $\mathcal{P}$ for the chain at fugacity $K_{j}$, and $\mathcal{P}_{j}$ and $\mathcal{P}_{j+1}$ the current conformations in the $j$-th and $(j+1)$-th chain, we accept the trial move (i.e. swap $\mathcal{P}_{j}$ and $\left.\mathcal{P}_{j+1}\right)$ with the acceptance rate

$$
a\left(\mathcal{P}_{j} \leftrightarrow \mathcal{P}_{j+1}\right)=\min \left(1, \frac{\pi_{j}\left(\mathcal{P}_{j+1}\right) \pi_{j+1}\left(\mathcal{P}_{j}\right)}{\pi_{j}\left(\mathcal{P}_{j}\right) \pi_{j+1}\left(\mathcal{P}_{j+1}\right)}\right) .
$$

Note that we do not need to compute the normalization of the limiting distributions (i.e. the partition functions) since these normalizations drop out in the ratio above.

The whole process is itself a Markov chain, which we can call a composite Markov chain. Since the underlying Markov chains are ergodic (for a fixed knot type), so is the composite Markov chain. Moreover the composite chain is in detailed balance since the "swap"-move as well as the moves in the underlying chains are. Consequently, the invariant limiting distribution is the product of the distributions of $m$ separate Markov chains at the temperatures $K_{1}<K_{2}<\ldots<K_{m}$.

One can clearly understand the advantage of this method by focusing on a particular value $K_{j}$. Every successful swap involving this chain and the chain $K_{j+1}$ corresponds to a big change in the configuration at this value $K_{j}$, so the correlation time at each $K_{j}$ is markedly reduced. Swaps move the system at a particular $K_{j}$ to new regions of the configuration space and therefore tend to eliminate problems of quasi-ergodicity. If two chains $K_{j}$ and $K_{j+1}$ are far apart, the changes in the configuration are likely to be large but eq.(17) suggests that bigger distances between the two selected chains lead to smaller acceptance rates for the swaps. In practice, the set of the values of $K$ is chosen so that successive values of $K$ are sufficiently close for swapping to occur rather frequently, and $K_{1}$ is small enough for convergence at $K_{1}$ to be rapid. All this requires that the total number $m$ of chains is sufficiently large. Hence the gain in convergence rate is obtained at only marginal cost in computer time.

In Table 2 we compare our estimates of the integrated autocorrelation time (given in units of sampling, i.e. per $10^{4}$ attempted local moves in our tests) of the average 
PSAP lengths, as a function of $K$, for the four different implementations of the algorithm: (1) the purely local algorithm using only elementary local moves, (2) the local + non-local algorithm, using both local and non-local moves, (3) the local MMC algorithm, using local moves only together with the MMC sampling and (4) the local + non local MMC algorithm, using local and non local moves together with the MMC sampling. In all cases, the integrated autocorrelation time for the polygon average length increases as $K$ approaches the critical value $K_{c}$, as expected. However, it is clear that both non-local moves and MMC swaps lead to a significant decrease in this integrated autocorrelation time.

\section{$5 \quad$ Results for $w=1$}

In this section, we focus on the model with $w=1$, i.e. we do not attach any particular weight to the crossings. A typical configuration with the topology of a trefoil knot is shown in figure 21.

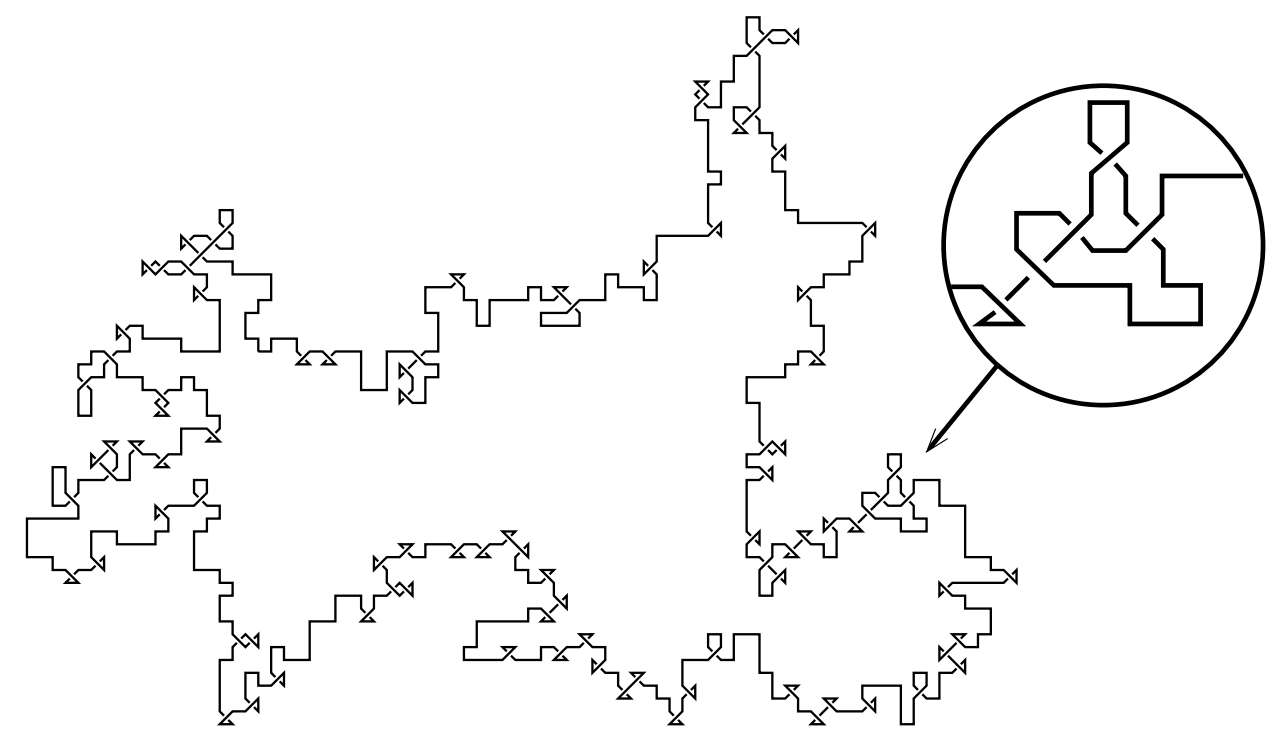

Figure 21: A typical configuration for the $3_{1}$ at $w=1$. The number of bonds is $n=906$. We have indicated the location of the knotted section.

\subsection{Connectivity $\mu$ and Entropic Exponent $\alpha$}

We present here our estimates for the connectivity constant $\mu$ of the PSAP and the entropic exponent $\alpha$ for $w=1$. These quantities are defined by the large $n$ behavior of the number $p_{n}(\tau)$ of configurations of fixed knot-topology $\tau$ with a fixed number 
$n$ of bonds, expected to be of the form

$$
p_{n}(\tau) \sim A(\tau)(\mu(\tau))^{n} n^{\alpha(\tau)-3}
$$

In particular we study the dependence of $\mu(\tau)$ and $\alpha(\tau)$ on the topology $\tau$ of the PSAP. A method to compute $\mu(\tau)$ and $\alpha(\tau)$ relies on the asymptotic behavior for the the average length $\langle n(\tau)\rangle$ of the PSAP for $K$ close to the critical fugacity $K_{c}(\tau)=$ $(\mu(\tau))^{-1}$, namely:

$$
\langle n(\tau)\rangle \approx \frac{[\alpha(\tau)+Q-2] K \mu(\tau)}{1-K \mu(\tau)}
$$

This behavior directly follows our choice of the statistical weight in eq.(II), which yields for $w=1$

$$
\langle n(\tau)\rangle=\frac{\sum_{n} n^{Q+1} p_{n}(\tau) K^{n}}{\sum_{n} n^{Q} p_{n}(\tau) K^{n}}
$$

Inserting eq.(18) directly leads to eq. (19). At leading order, we can use eq.(19) to approximate $1 /\langle n(\tau)\rangle$ as:

$$
\frac{1}{\langle n(\tau)\rangle} \approx \frac{1-K \mu(\tau)}{[\alpha(\tau)+Q-2] K \mu(\tau)}=\frac{1}{[\alpha(\tau)+Q-2] K \mu(\tau)}-\frac{1}{\alpha(\tau)+Q-2} .
$$

An estimate of $\mu(\tau)$ and $\alpha(\tau)$ can therefore be obtained by a linear extrapolation of $1 /\langle n(\tau)\rangle$ as a function of $1 / K$ for $1 /\langle n(\tau)\rangle \rightarrow 0$.

In figure 22 we have plotted $1 /\langle n(\tau)\rangle$ as a function of $1 / K$ for the topology of the unknot $(\emptyset)$, that of the trefoil knot $\left(3_{1}\right)$, that of the figure eight knot $\left(4_{1}\right)$, that of the composite knot made of two trefoils $\left(3_{1} \# 3_{1}\right)$ and that of the composite knot made of a trefoil and a figure eight $\left(3_{1} \# 4_{1}\right)$. These data are obtained with $Q=2$. We note that as the complexity of the topology increases, the corrections to the linear scaling (21) become more and more important, but for $K$ sufficiently close to the critical value $K_{c}(\tau)=\mu(\tau)^{-1}$, a linear behavior is indeed obtained. By extrapolating the data, using the linear fit of equation (21), we obtain the following estimates:

$$
\begin{aligned}
\mu(\emptyset) & =3.254 \pm 0.01, \\
\mu\left(3_{1}\right) & =3.250 \pm 0.06, \\
\mu\left(4_{1}\right) & =3.255 \pm 0.07, \\
\mu\left(3_{1} \# 3_{1}\right) & =3.249 \pm 0.13 \\
\mu\left(3_{1} \# 4_{1}\right) & =3.261 \pm 0.15
\end{aligned}
$$

These values coincide up to the second decimal, and it seems reasonable to assume that they are indeed all equal. In fact, since they were measured from completely independent simulations, we can take their average to estimate the growth constant of PSAP of a fixed knot type: we obtain $\mu(\tau)=\mu_{1}=3.251 \pm 0.002$ (95\% confidence interval) for PSAP's with arbitrary fixed topology $\tau$. From the same linear fit an estimate of the entropic exponent $\alpha$ can also be obtained:

$$
\alpha(\emptyset)=0.58 \pm 0.07
$$




$$
\begin{aligned}
\alpha\left(3_{1}\right) & =2.10 \pm 0.08, \\
\alpha\left(4_{1}\right) & =2.13 \pm 0.10, \\
\alpha\left(3_{1} \# 3_{1}\right) & =3.91 \pm 0.24, \\
\alpha\left(3_{1} \# 4_{1}\right) & =4.05 \pm 0.25 .
\end{aligned}
$$

In contrast with $\mu$, the exponent $\alpha$ depends clearly on the topology. However, this dependence appears to be weak in the sense that $\alpha$ depends only on how many primary knots form the knot under consideration and not on the precise nature of these knots, hence $\alpha\left(3_{1}\right)=\alpha\left(4_{1}\right)$ and $\alpha\left(3_{1} \# 3_{1}\right)=\alpha\left(3_{1} \# 4_{1}\right)$ within numerical uncertainties. Such a result was already found in [10, 11] for three-dimensional knotted polygons. There however, it was found that $\alpha(\tau)$ increases by one for each added primary knot, leading to $\alpha(\tau)=\alpha(\emptyset)+N_{f}$ where $\tau$ is a knot with $N_{f}$ prime factors. This formula does not seem to hold in our case.

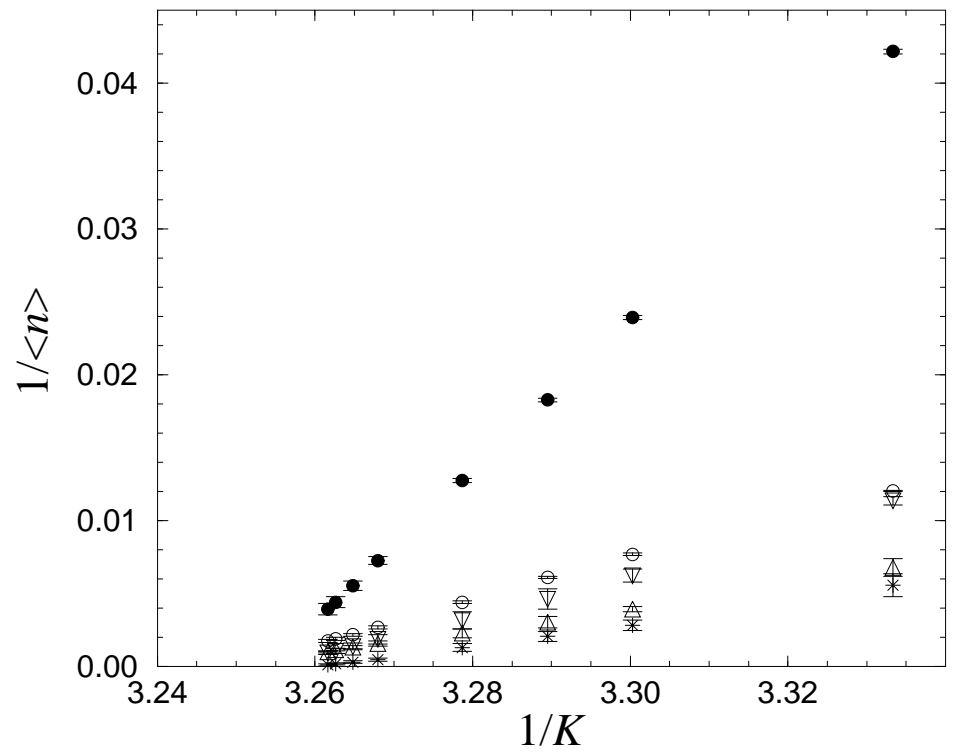

Figure 22: Plot of $1 /\langle n\rangle$ as a function of $1 / K$ for the unknot $(\bullet)$, the trefoil (o), the figure eight $(\nabla)$, the composite knot $3_{1} \# 3_{1}(\Delta)$ and the composite knot $3_{1} \# 4_{1}(*)$.

Another way to estimate $\mu$ and $\alpha$ relies on the maximum likelihood method as suggested in [21]. In this case, we assume that for $n>n_{\min }$ the relation

$$
p_{n}=\mu^{n} n^{\alpha-3}(1+a / n)
$$

holds, where $a$ is a free parameter that we can vary to mimic a correction to scaling. By varying $n_{\min }$ and $a$ we can probe the sensitivity of our estimates to scaling. We generally find a region of minimum sensitivity from which we extract the following estimates:

$$
\mu(\emptyset)=3.254 \pm 0.005
$$




$$
\begin{aligned}
\mu\left(3_{1}\right) & =3.251 \pm 0.07 \\
\mu\left(4_{1}\right) & =3.253 \pm 0.09 \\
\mu\left(3_{1} \# 3_{1}\right) & =3.245 \pm 0.10 \\
\mu\left(3_{1} \# 4_{1}\right) & =3.255 \pm 0.11 .
\end{aligned}
$$

and for the entropic exponents:

$$
\begin{aligned}
\alpha(\emptyset) & =0.58 \pm 0.03, \\
\alpha\left(3_{1}\right) & =1.86 \pm 0.06, \\
\alpha\left(4_{1}\right) & =1.95 \pm 0.08 \\
\alpha\left(3_{1} \# 3_{1}\right) & =4.20 \pm 0.15 \\
\alpha\left(3_{1} \# 4_{1}\right) & =3.90 \pm 0.20 .
\end{aligned}
$$

All these results are in agreement with the estimates (23) and (24).

\subsection{The Metric Exponent $\nu$}

For the mean square radius of gyration, we assume the large $n$ behavior

$$
\left\langle R^{2}(\tau)\right\rangle_{n}=A_{\nu}(\tau) n^{2 \nu(\tau)}
$$

where the average is performed over configurations with fixed $n$. In order to estimate $\nu$ from our data in the grand-canonical ensemble, we compute the expected value of the mean square radius of gyration,

$$
\left\langle R^{2}(\tau)\right\rangle=\frac{\sum_{n}\left\langle R^{2}(\tau)\right\rangle_{n} n^{Q} p_{n}(\tau) K^{n}}{\sum_{n} n^{Q} p_{n}(\tau) K^{n}}
$$

Using the asymptotic forms (18) and (27), we get the following approximation for $\left\langle R^{2}(\tau)\right\rangle$ close to the critical $K_{c}$ :

$$
\left\langle R^{2}(\tau)\right\rangle \simeq h(\tau)(1-K \mu(\tau))^{-2 \nu(\tau)}
$$

or equivalently, using eq.(19), the approximation:

$$
\left\langle R^{2}(\tau)\right\rangle \simeq h^{\prime}(\tau)\langle n(\tau)\rangle^{2 \nu(\tau)}
$$

where we now have a relation between $\left\langle R^{2}(\tau)\right\rangle$ and $\langle n(\tau)\rangle$. A log-log fit of our data to equation (30), where $K$ takes a different value for each chain in our multiple Markov chain Monte Carlo, can be used to estimate $\nu$ for each knot type. Our best estimates are

$$
\begin{aligned}
\nu(\emptyset) & =0.747 \pm 0.005, \\
\nu\left(3_{1}\right) & =0.758 \pm 0.010, \\
\nu\left(4_{1}\right) & =0.748 \pm 0.010, \\
\nu\left(3_{1} \# 3_{1}\right) & =0.760 \pm 0.020, \\
\nu\left(3_{1} \# 4_{1}\right) & =0.720 \pm 0.040 .
\end{aligned}
$$


Within the stated error bars, the results above are all identical and consistent with the exact value $\nu_{\mathrm{SAW}}=3 / 4$ [23] for self-avoiding walks or self-avoiding polygons in $d=2$. This seems to indicate that although polygons in our model are not strictly selfavoiding since crossings are allowed for $w=1$, the presence of these crossings limited to diagonal edges does not modify the scaling behavior of the radius of gyration.

\section{Results for $w<<1$}

It is interesting to consider our model in the limit $w \rightarrow 0$ where crossings become forbidden. At $w=0$, our model coincides with that of a strictly self-avoiding polygon on the square lattice. Still, we can start from a configuration with a non-trivial knot topology $\tau$ (say that of the trefoil knot) with its minimal number $c_{\min }(\tau)$ of crossings $\left(c_{\min }\left(3_{1}\right)=3\right.$ for the trefoil) and look at the limit $w \rightarrow 0$ of $G_{\tau}(Q, K, w) / w^{c_{\min }(\tau)}$. In this case, we explore the phase space of all the configuration of knot type $\tau$ and with the minimal number of crossings $c_{\min }(\tau)$. This limit is the "minimal" extension of the strictly self-avoiding model to include a non-trivial topology. In this limit, we of course expect the model to be in the universality class of strictly self-avoiding walks.

\subsection{Results for $\mu$ and $\alpha$}

In practice, the easiest way to study the $w \rightarrow 0$ limit is simply to fix in our simulation a very small value of $w$. The results presented here have been obtained with the value of the crossing fugacity $w$ fixed to $w=0.0001$. Here again we define the connectivity constant $\mu$ and the entropic exponent $\alpha$ for the asymptotic behavior of the number $p_{n}^{(0)}(\tau)$ of configurations of polygons in the topology $\tau$, with $n$ bonds and with the minimal number $c_{\min }(\tau)$ of crossings compatible with $\tau$. As for the case $w=1$, we can estimate $\mu$ and $\alpha$ by plotting $1 /\langle n(\tau)\rangle$ as a function of $1 / K$ and by making a linear extrapolation for $1 /\langle n(\tau)\rangle \rightarrow 0$.

For $\mu$ we obtain the following estimates:

$$
\begin{aligned}
\mu(\emptyset) & =2.640 \pm 0.01, \\
\mu\left(3_{1}\right) & =2.640 \pm 0.01, \\
\mu\left(4_{1}\right) & =2.639 \pm 0.02, \\
\mu\left(3_{1} \# 3_{1}\right) & =2.641 \pm 0.05, \\
\mu\left(3_{1} \# 4_{1}\right) & =2.645 \pm 0.08 .
\end{aligned}
$$

These values coincide to the second decimal place, and it seems reasonable to assume that they are indeed all equal. It is interesting to notice that these estimates of $\mu(\tau)$ are a good upper bound for the best available estimates of the connectivity constant for self-avoiding polygons in the $d=2$ square lattice $\left(\mu_{0}=2.63815\right)$ [22].

For the entropic exponents we obtain:

$$
\begin{aligned}
\alpha(\emptyset) & =0.503 \pm 0.040 \\
\alpha\left(3_{1}\right) & =2.24 \pm 0.10
\end{aligned}
$$




$$
\begin{aligned}
\alpha\left(4_{1}\right) & =2.28 \pm 0.10 \\
\alpha\left(3_{1} \# 3_{1}\right) & =3.2 \pm 0.3 \\
\alpha\left(3_{1} \# 4_{1}\right) & =3.3 \pm 0.5 .
\end{aligned}
$$

As for the case $w=1$, we again see a dependence of $\alpha$ on the number of prime factors forming the knot. We note however that the values of $\alpha$ for a fixed knot type are different in this limit $(w \rightarrow 0)$ from the $w=1$ values. For the unknot, we recover the exact value $\alpha_{\mathrm{SAW}}=1 / 2$ [23]. This is expected since strictly self-avoiding rings in two dimensions cannot form knots, thus the usual $\alpha$ exponent derived form field theory corresponds to fixing the topology to that of the unknot rather than summing over all topologies as in three dimensions.

\subsection{The Metric Exponent $\nu$}

As for the case $w=1$, we have evaluated the exponent $\nu$ in the limit $w \rightarrow 0$ from the relation of eq. 30 between the average radius of gyration $\left\langle R^{2}(\tau)\right\rangle$ and the average number of bonds $\langle n(\tau)\rangle$. Our estimates are

$$
\begin{aligned}
\nu(\emptyset) & =0.748 \pm 0.015, \\
\nu\left(3_{1}\right) & =0.753 \pm 0.010, \\
\nu\left(4_{1}\right) & =0.749 \pm 0.011 \\
\nu\left(3_{1} \# 3_{1}\right) & =0.736 \pm 0.015 \\
\nu\left(3_{1} \# 4_{1}\right) & =0.742 \pm 0.020 .
\end{aligned}
$$

We thus obtain an exponent $\nu$ independent of the knot-type and in perfect agreement with the exact value $\nu_{\mathrm{SAW}}=3 / 4$ for self-avoiding walks in two dimensions.

\subsection{Statistics of Arc Lengths: Localization of Knots.}

One advantage of taking this limit $w \rightarrow 0$ is that, by preventing the creation of spurious crossings, we have a fixed minimal number $c_{\min }(\tau)$ of crossings which we can easily localize along the PSAP. In particular, we can study the typical "size" of the knot in a given topology and see for instance if primary knots tend to be localized on a small number of bonds or prefer to extend over the whole PSAP. A simple way to analyze this phenomenon is to study the distribution on the lengths of the arcs between consecutive crossings.

In the case of a trefoil knot, we have exactly $c_{\min }\left(3_{1}\right)=3$ crossings which separate exactly 6 arcs along the PSAP. For each configuration with $n$ bonds, we can classify these arcs in order of decreasing arc length $l_{i}=\lambda_{i} n$ with $\lambda_{1} \geq \lambda_{2} \geq \cdots \geq \lambda_{6}$ and $\sum_{i=1}^{6} \lambda_{i}=1$. Here $\lambda_{i}$ is thus the fraction of the total length $n$ inside the $i$-th largest arc.

In figure 23, we have plotted the fraction of length occupied by the two largest arcs for a trefoil knot, as a function of $K$. We see that as $K \rightarrow K_{c}(\langle n\rangle \rightarrow \infty), \lambda_{1} \rightarrow 1$ while $\lambda_{2}$, and thus all the other $\lambda_{i}$ for $i>2$, tend to zero. This result is consistent 


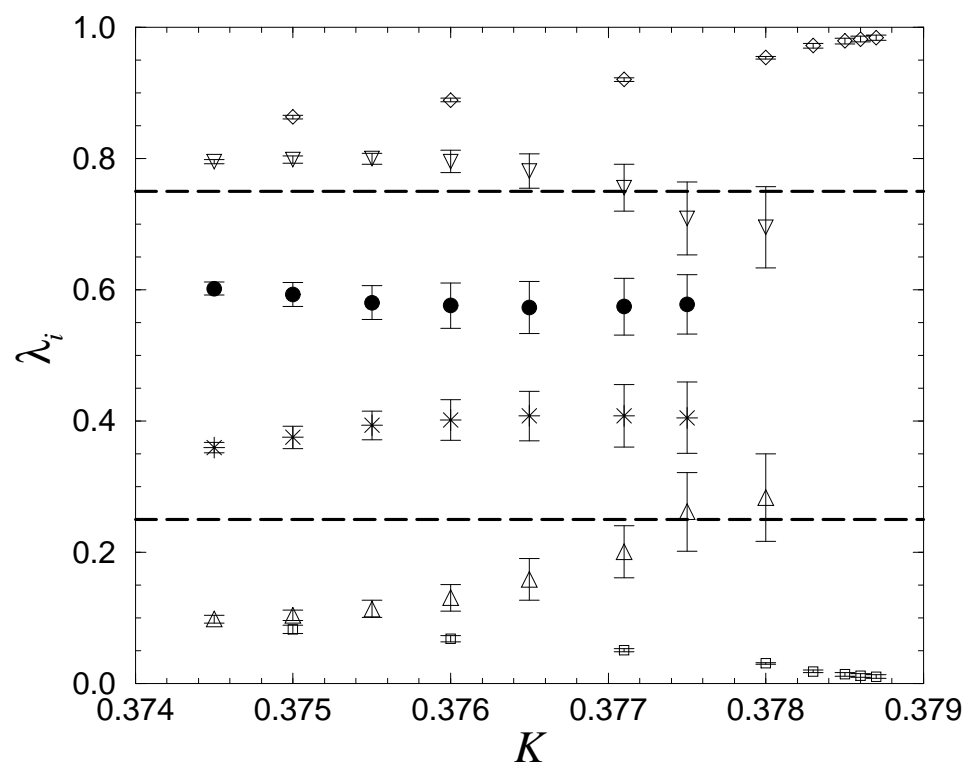

Figure 23: Average length fraction $\lambda_{1}$ and $\lambda_{2}$ of the two largest arcs along the PSAP as a function of $K$ for the trefoil $\left(\lambda_{1} \rightarrow \diamond, \lambda_{2} \rightarrow \square\right)$, the composite knot $3_{1} \# 3_{1}$ ( $\nabla$ and $\Delta)$ and the composite knot $33_{1} \# 4_{1}(\bullet$ and $*)$. The dashed lines indicate the values $3 / 4$ and $1 / 4$ expected for two strictly localized knots moving freely along the PSAP.

with a localized knot which takes place over a number a bonds which is either finite, or at least growth less rapidly than $n$. This result of localization is corroborated by a direct visualization of a typical configuration, as shown in figure 24.

Another interesting case is that of composite knots $3_{1} \# 3_{1}$ and $3_{1} \# 4_{1}$, which have respectively $c_{\min }\left(3_{1} \# 3_{1}\right)=6$ and $c_{\min }\left(3_{1} \# 4_{1}\right)=7$. These knots are thus made respectively of exactly 12 and 14 arcs. We have plotted on figure 23 the proportions of the total length $n$ occupied by the two largest arcs in both cases. Although the statistical errors are rather important when $K \rightarrow K_{c}$, we see in each case that these proportions add up to one, with the proportion occupied by all the smaller arcs tending to zero. This result is consistent with a picture of the composite knot as made of two perfectly localized primary factors. The localization of the two primary knots is also directly visible on a typical configuration of the PSAP (see figure 25). If we assume that these localized factors move freely along the PSAP and interact only locally by preventing to pass through one another (which would require creating additional crossings), we should expect that the proportions tend to $3 / 4$ and $1 / 4$. Indeed, for two points at random drawn on a loop of size $n$, the largest arc between them has average length $(1 / n) \int_{0}^{n} d x \max (x, n-x)=(3 n / 4)$ and the complementary arc has thus average length $n / 4$. In practice, we observe a value a $\lambda_{1}<3 / 4$ and $\lambda_{2}>1 / 4$, which tends to indicate that the primary knots repel each other. This repulsion might however be a finite size effect. Indeed the deviation from the expected asymptotic values $3 / 4$ and $1 / 4$ is larger for the $3_{1} \# 4_{1}$ knot than for the $3_{1} \# 3_{1}$ knot 


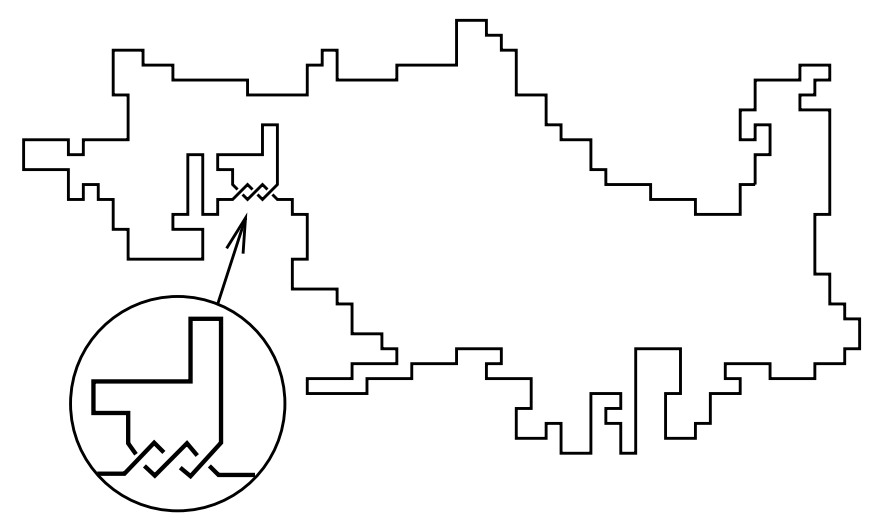

Figure 24: A typical configuration for the trefoil knot $\left(3_{1}\right)$ at $w \sim 0$. The number of bonds is $n=312$. We have indicated the location of the knotted section.

as the $4_{1}$ prime factor typically requires more bonds to be formed.

\section{$7 \quad$ Results for $w>1$}

Up to now, we have studied only the case $w=1$ where no fugacity is associated to the number of crossings and the case $w \rightarrow 0$ where we have a minimal number of crossings compatible with the knot topology at hand. Although we obtained different values for the (non universal) connectivity constant $\mu$, we found in both cases a value of $\nu$ compatible with the exact result $\nu_{\mathrm{SAW}}=3 / 4$ in two dimensions, which suggest that these two cases are in the universality class of self-avoiding walks, and so is probably any case of the model with a value of $w$ between 0 and 1 . In this section, we will rather be interested in the behavior of the model for $w>1$.

\subsection{Connectivity $\mu$}

Let us first concentrate on the connectivity constant $\mu$. If we denote by $p_{n, c}(\tau)$ the number of configurations of PSAP with $n$ bonds, $c$ crossings and the knot topology $\tau$, we can introduce the canonical partition function

$$
p_{n}(w, \tau)=\sum_{c \geq c_{\min (\tau)}} p_{n, c}(\tau) w^{c-c_{\min }(\tau)}
$$

For $w=1$, we recover the number $p_{n}(\tau)$ of configurations with knot-topology $\tau$ and with $n$ bonds, irrespectively of their number of crossings. For $w=0$, we recover the number $p_{n}^{(0)}(\tau)$ of configurations with topology $\tau$, number of bonds $n$, and with the minimal number of crossings $c_{\min }(\tau)$.

We define $\mu(w, \tau)$ and $\alpha(w, \tau)$ by the large $n$ behavior

$$
p_{n}(w, \tau) \sim A(w, \tau) \mu(w, \tau)^{n} n^{\alpha(w, \tau)-3}
$$




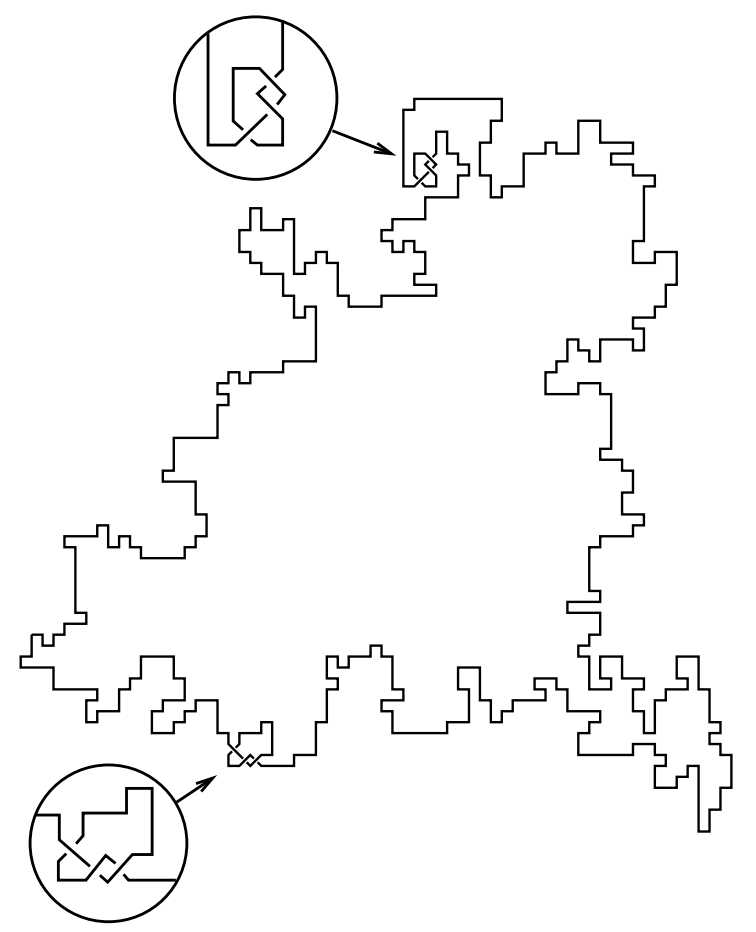

Figure 25: A typical configuration for the composite knot $3_{1} \# 3_{1}$ at $w \sim 0$. We have indicated the location of the two primary knots.

In the grand canonical ensemble, we have

$$
G_{\tau}(Q, K, w) / w^{c_{\min }(\tau)}=\sum_{n} p_{n}(w, \tau) K^{n} n^{Q}
$$

hence we can again relate $\mu(w, \tau)$ to the inverse of the critical value $K_{c}(w)$ of $K$ for which $\langle n(w, \tau)\rangle$ diverges.

Before we present our numerical results, let us first establish a lower and an upper bound for $\mu(w)$.

Lower bound for $w>1$ : The canonical partition function $p_{n}(w, \tau)$ is clearly bounded from below by the contribution $w^{c}$ of any acceptable configuration in the given knot-topology class. Since $w>1$, we can obtain a larger lower bound by selecting a configuration with the maximum number of crossings. To obtain such a configuration, we start from any particular realization of the knot, as compact as possible. It will have, say, $n_{0}$ bonds and $c_{0}$ crossings. On any outermost bond, we can start to grow a linear sequence of crossings, such as the one depicted in figure 26. Note that we can arbitrarily choose any of the two possible crossings for each new crossing added in linear piece. The addition of this linear sequence clearly does not modify the topology of the knot. If $n$ is the total number of bonds in the configuration, we get a total number of crossings equal to $c_{0}+\left(n-n_{0}\right) / 2$ for any of the $2^{\left(n-n_{0}\right) / 2}$ such configurations. We thus get

$$
p_{n}(w, \tau)>2^{-\frac{n_{0}}{2}} w^{c_{0}-\frac{n_{0}}{2}-c_{\min }(\tau)} \times(2 w)^{\frac{n}{2}}
$$




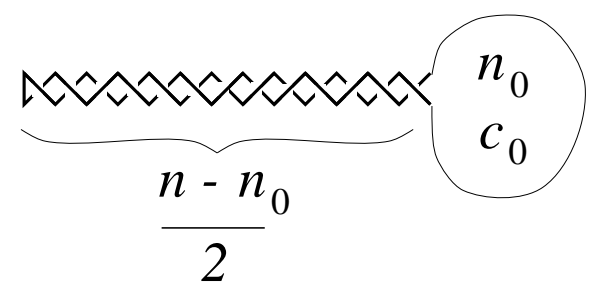

Figure 26: The growing of a linear sequence of crossings from a compact knot.

In the large $n$ limit, this directly leads to the lower bound

$$
\mu(w) \geq \sqrt{2 w}
$$

Upper bound for $w>1$ : It is clear that any configuration with $n$ bonds can have at most $n / 2$ crossings, irrespectively of its knot type. We thus get for $w>1$

$$
p_{n}(w, \tau)<p_{n}(\tau) w^{\frac{n}{2}}
$$

This directly leads to

$$
\mu(w, \tau) \leq \mu_{1} \sqrt{w}
$$

From eqs.(39) and (41), we can deduce that at large $w, \mu(w)$ behaves like

$$
\mu(w, \tau) \stackrel{w \rightarrow \infty}{\sim} \lambda \sqrt{w}
$$

with $\sqrt{2} \leq \lambda \leq \mu_{1} \sim 3.251$.

To complete our analysis, we can also give lower and upper bounds on the case $0<w<1$. In this case, it is clear that

$$
p_{n}^{(0)}(\tau)<p_{n}(w, \tau)<p_{n}(\tau)
$$

and therefore

$$
2.638 \sim \mu_{0} \leq \mu(w, \tau) \leq \mu_{1} \sim 3.251
$$

Figure 27 presents our results for $\mu(w)$ together with the above lower and upper bounds. At large $w$, we confirm the behavior of eq.(42) with $\lambda \simeq 1.46$ close to the lower bound $\sqrt{2}$.

\subsection{The Metric Exponent $\nu$ : Branched Polymer Behavior}

We have also estimated the metric exponent $\nu$ for different values of $w>1$. Our best estimates are

$$
\begin{aligned}
& \nu(w=2)=0.668 \pm 0.008, \\
& \nu(w=3)=0.665 \pm 0.007
\end{aligned}
$$




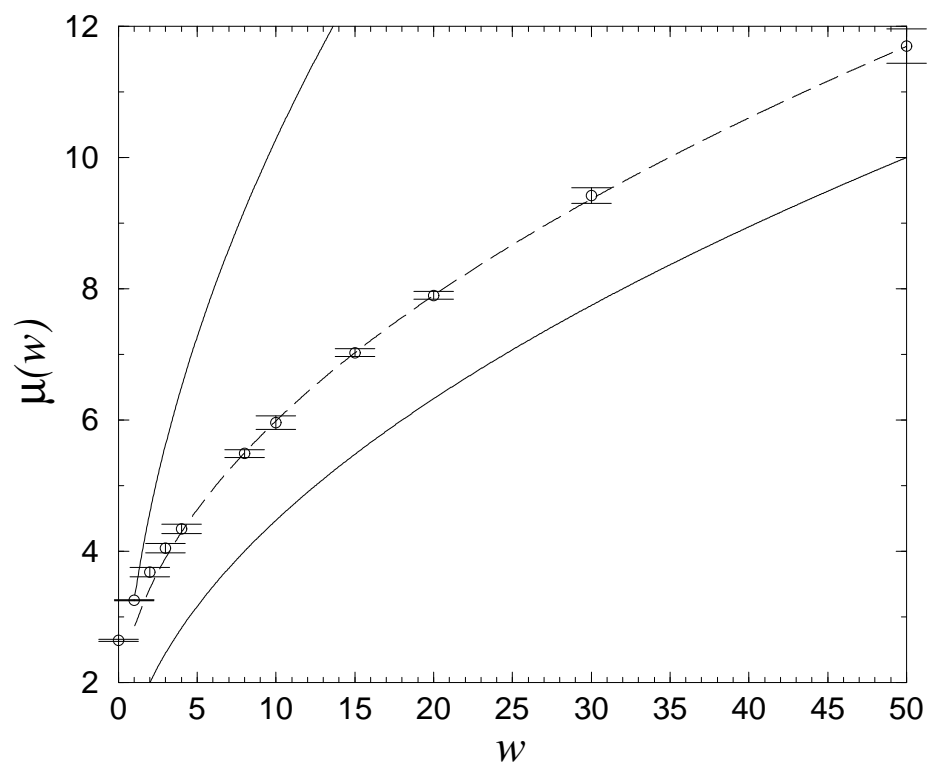

Figure 27: Estimate of $\mu$ as a function of the crossing fugacity $w$; the solid lines correspond respectively to the curves $\mu=3.251 w^{\frac{1}{2}}$ (upper bound) and $\mu=(2 w)^{\frac{1}{2}}$ (lower bound). The dashed line corresponds to the fit $\mu=1.46\left(w^{\frac{1}{2}}-1\right)+2.86$.

$$
\begin{gathered}
\nu(w=4)=0.645 \pm 0.008, \\
\nu(w=10)=0.634 \pm 0.010, \\
\nu(w=15)=0.630 \pm 0.011, \\
\nu(w=20)=0.625 \pm 0.012, \\
\nu(w=30)=0.620 \pm 0.015, \\
\nu(w=50)=0.624 \pm 0.020,
\end{gathered}
$$

These values are plotted in figure 28, together with the values estimated for $w \rightarrow 0$ and $w=1$. Within the error bars, we distinguish two values for $\nu$. For $w \leq 1$, our data are consistent with the value $\nu_{\mathrm{SAW}}=3 / 4$ of self-avoiding walks. For $w>1$ however, our estimates are far below this value and approximately located around the value $\nu_{\mathrm{BP}} \sim$ 0.64 obtained for branched polymers in $D=2$ [24, 25]. At large $w$, the branched polymer picture is corroborated by looking at a typical configuration, as the one depicted in figure 29. Such configurations are naturally selected at large $w$ since long one-dimensional coils maximize the number of crossings and are thus energetically favored, while branching points, occuring at a finite energy cost, are favored for entropic reasons. From our data, a transition from the self-avoiding behavior to a branched polymer type statistics seems to occur at $w=1$. For $w<1$, crossings are penalized and play a marginal role without changing the asymptotic behavior of the polymer, which can be considered as self-avoiding. On the contrary, for $w>1$, a finite density of crossings is present in a typical configuration, which degenerates into a branched polymer conformation. At the transition point $w=1$, we find 


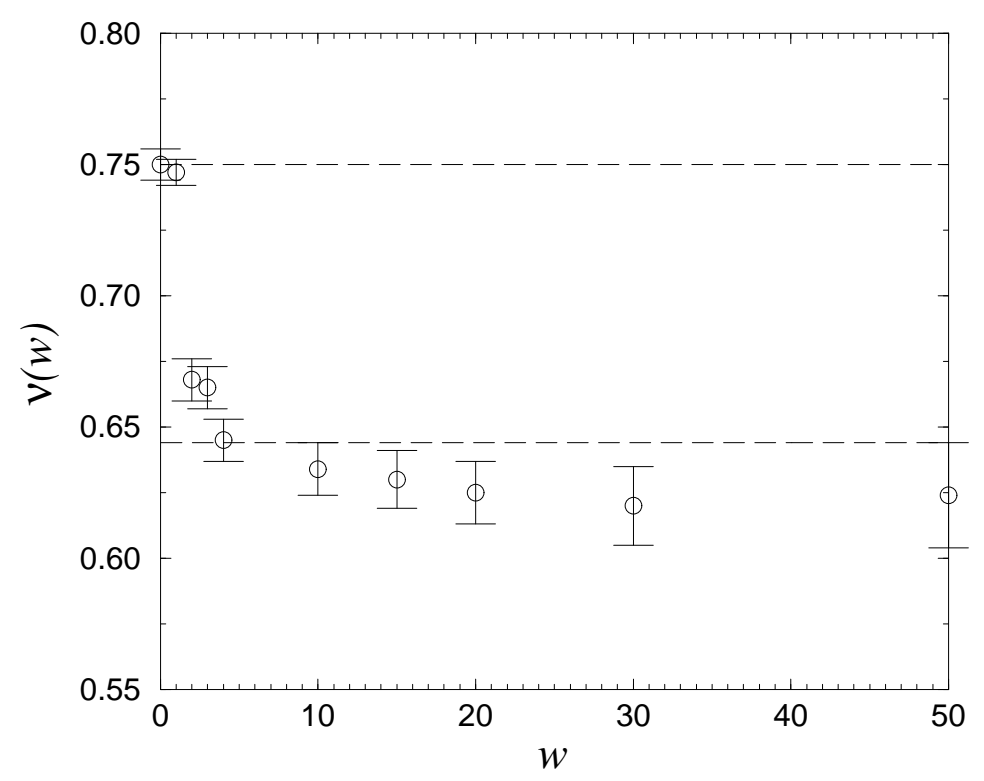

Figure 28: The exponent $\nu$ as a function of $w$. The dashed lines indicate the values of $\nu$ for self-avoiding walks and branched polymers.

occasionally interesting configurations showing a phase separation between a phase rich in crossings and a phase with few crossings (see figure 30). In this case, the largest of the largest value $\nu=\nu_{\text {SAW }}$ is observed. Finally, let us note that a similar transition from a self-avoiding behavior to a branched polymer behavior has already been observed in an interacting two-tolerant trail model on the square lattice [26].

\section{Conclusions}

In this paper, we introduced a two-dimensional lattice model of Projected SelfAvoiding Polygons, describing two-dimensional knotted polymers rings with a fixed knot topology. We made a numerical study of the model using a Monte Carlo algorithm based on both local and non-local moves which preserve the knot topology of the polygon, together with a Multiple Markov Chain procedure. In the absence of a rigorous proof of the ergodicity of our algorithm within a fixed knot type, we gave strong arguments in favor of this ergodicity, together with the guidelines of a possible proof. We presented our numerical results for the connectivity constant $\mu$ and the critical exponents $\nu$ and $\alpha$ for various topologies and various values of the fugacity $w$ associated to the number of crossings in the projected polygon. The connectivity $\mu$ is found to depend only on this fugacity $w$ and not on the particular knot topology at hand. This is somewhat expected since $\mu$ typically measures a local property which is not affected by a global constraint such as fixing the knot topology. By measuring the radius of gyration of the polygon, we observe only two possible values for the exponent $\nu$ : a self-avoiding value $3 / 4$ found at low $w \leq 1$ irrespectively of the knot 


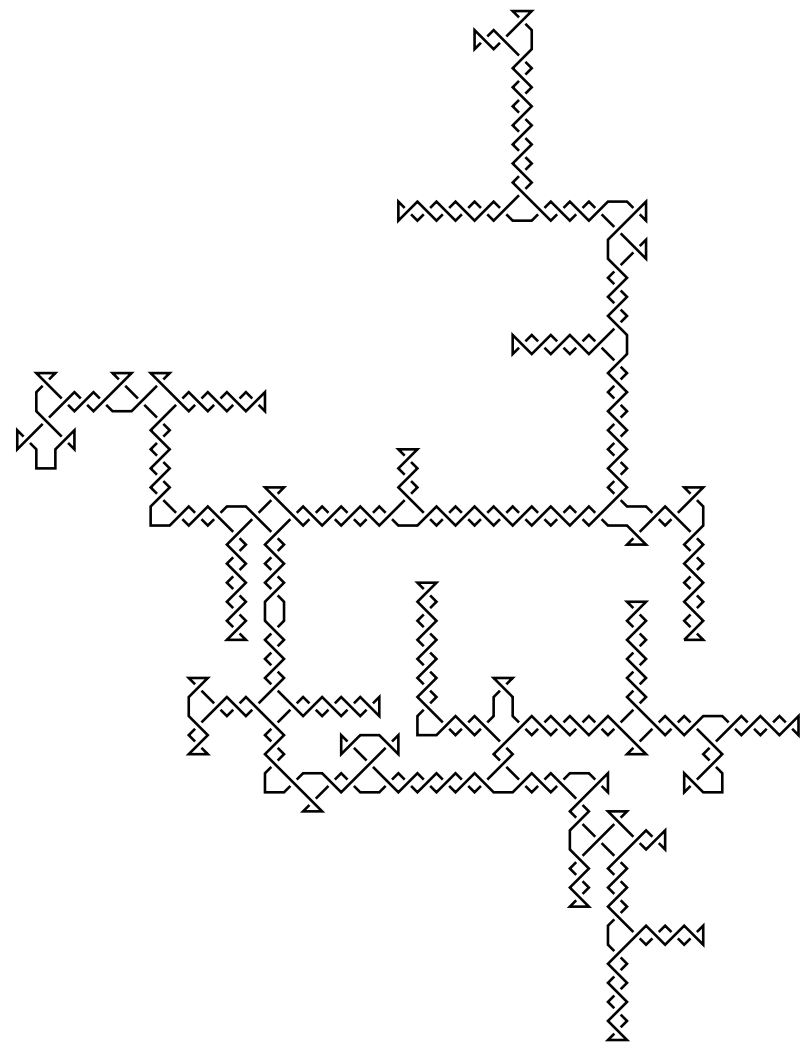

Figure 29: A typical configuration for the unknot $(\emptyset)$ at $w=30$.

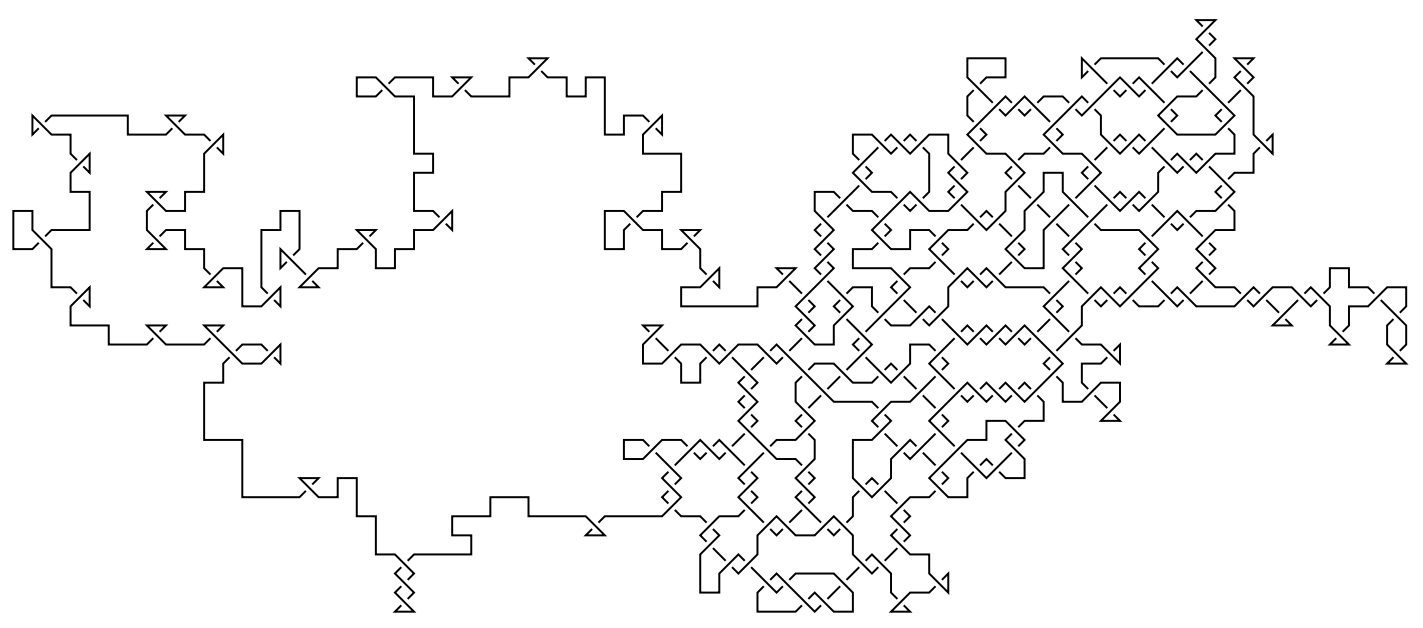

Figure 30: A configuration for the unknot at $w=1$, with $n=1250$ bonds. One clearly sees two domains with very different densities of crossings, one being sparse while the other is dense. 
type, and a large branched polymer value $\simeq 0.64$ found at large $w>1$ for all knot topologies. Our data suggest that the transition between the two regimes occurs precisely at $w=1$. Finally, at fixed $w$, the exponent $\alpha$ for the number of accessible configurations is found to depend only on the number of prime factors forming the knot. A weak $w$ dependence is also observed.

One nice feature of our two-dimensional model is the possibility to have more specific characterizations of the polygon conformations, such as the number of crossings or the arc lengths between successive crossings along the chain. This allowed us in particular to measure the typical extension of a knot inside the polygon. In the limit $w \rightarrow 0$, we found for instance that the prime factors in a knotted polygon are localized and weakly interacting.

Other applications of this model can be imagined, such as a statistics of the knot type of randomly drawn polygons. For an arbitrary polygon, the determination of the knot topology could be simply achieved in principle by imposing a very low value of $w$ and letting the system evolve toward a configuration with a minimal number of crossings in the projection. In particular, it would be interesting to understand how the knot complexity increases with the number of monomers in the chain. 


\section{References}

[1] P. G. de Gennes, Exponents for the excluded volume problem as derived by the Wilson method Phys. Lett. A 38 (1972) 339-340.

[2] D.W. Sumners and S.G. Whittington, Knots in self-avoiding walks, J. Phys. A: Math. Gen. 21 (1988), 1689-1694.

[3] N. Pippenger, Knots in random walks, Discrete Applied Math. 25 (1989), 273-278.

[4] V.V. Ribenkov, N.R. Cozzarelli and A.V. Vologodskii, Probability of DNA knotting and the effective diameter of the DNA double helix, Proc. Natl. Acad. Sci. U.S.A. 90 (1993), 5307-5311.

[5] S.Y. Shaw and J.C. Wang, Knotting of a DNA chain during ring closure, Science 260 (1993) 533-536.

[6] E. J. Janse van Rensburg and S.G. Whittington, The dimensions of knotted polygons, J. Phys. A: Math. Gen. 24 (1991), 3935-3948.

[7] T. Deguchi and K. Tsurusaki, Topology of closed random polygons, J. Phys. Soc. Japan 62 (1993), 1411-1414.

[8] T. Deguchi and K. Tsurusaki, Statistical study of random knotting using Vasiliev invariants, in Random Knotting and Linking ed K C Millett and D. W. Sumners (Singapore: World Scientific) (1994), 89-121.

[9] S. Quake, Topological effects of knots in polymers, Phys. Rev. Lett. 73 (1994), 3317-3320.

[10] E. Orlandini, M.C. Tesi, E.J. Janse van Rensburg and S.G. Whittington, Entropic exponents of lattice polygons with specified knot type,

[11] E. Orlandini, M.C. Tesi, E.J. Janse van Rensburg and S.G. Whittington, Asymptotics of knotted lattice polygons, J. Phys A: Math. Gen. 31 (1998), 5953-5967.

[12] For an introduction to knot theory, see for instance C.C. Adams, The Knot Book: an elementary introduction to the mathematical theory of knots, Ed. W. H. Freeman and company, New York (1994).

[13] B. Berg and D. Foester, Random paths and random surfaces on a digital computer, Phys. Lett. 106B (1981), 323-326.

[14] C. Aragao de Carvalho and S. Caracciolo, A new Monte Carlo approach to the critical properties of self-avoiding random walks, Journal de Physique 44 (1983), 323-331.

[15] C. Aragao de Carvalho, S. Caracciolo and J. Fröhlich, Polymers and $g|\phi|^{4}$-theory in four dimensions, Nucl. Phys. B [FS7] 215 (1983), 209-248. 
[16] A.D. Sokal and L.E. Thomas, Exponential convergence to equilibrium for a class of random walk models, J. Stat. Phys. 54 (1989), 797-828.

[17] S. Caracciolo, A. Pellissetto and A.D. Sokal, Nonlocal Monte Carlo algorithm for self-avoiding walks with fixed endpoints, J. Stat. Phys (1990), 1-53.

[18] C.J. Geyer, Markov chain Monte Carlo maximum likelihood, in Computing Science and Statistics: Proceedings of the 23rd Symposium on the Interface, ed E M Keramidas (Interface Foundation) (1991), 156-163.

[19] M.C. Tesi, E.J. Janse van Rensburg, E. Orlandini and S.G. Whittington, Monte Carlo study of the interacting self-avoiding walk model, J. Stat. Phys. 82 (1996), 155-181.

[20] E. Orlandini, Monte Carlo study of polymer systems by Multiple Markov Chain method, IMA Proc. Work 7 (1996).

[21] A. Berretti and A. Sokal, , J. Stat. Phys. 40 (1985), 1689-1694.

[22] S. Caracciolo, A. Pellissetto and A.D. Sokal, Monte Carlo test of hyperscaling relation for the two-dimensional self-avoiding walk:II, J. Phys. A: Math. Gen. 23 (1990), 4509-4517.

[23] B. Nienhuis, Exact critical point and critical exponents of $O(n)$ models in two dimensions Phys. Rev. Lett. 49 (1982), 1062-1065.

[24] B. Derrida and L. De Seze, Application of the phenomenological renormalization to percolation and lattice animals in dimension 2, J. Physique 43 (1982), 475-483.

[25] E.J. Janse van Rensburg and N. Madras, A non-local Monte Carlo algorithm for lattice trees J. Phys. A: Math. Gen. 25 (1992) 303-333.

[26] E. Orlandini, F. Seno, A.L. Stella and M.C. Tesi, Collapse from linear to branched polymer behavior, Phys. Rev. Lett. 68 (1992) 488-491, see also L. Turban, Generalised self-avoiding walk, J. Phys. A: Math. Gen. 16 (1983) L643-646. 